\title{
Ultrashort electron pulses for diffraction, crystallography and microscopy: theoretical and experimental resolutions
}

\author{
Andreas Gahlmann, Sang Tae Park and Ahmed H. Zewail* \\ Received 6th February 2008, Accepted 6th March 2008 \\ First published as an Advance Article on the web 31st March 2008 \\ DOI: $10.1039 / \mathbf{b 8 0 2 1 3 6 h}$
}

Pulsed electron beams allow for the direct atomic-scale observation of structures with femtosecond to picosecond temporal resolution in a variety of fields ranging from materials science to chemistry and biology, and from the condensed phase to the gas phase. Motivated by recent developments in ultrafast electron diffraction and imaging techniques, we present here a comprehensive account of the fundamental processes involved in electron pulse propagation, and make comparisons with experimental results. The electron pulse, as an ensemble of charged particles, travels under the influence of the space-charge effect and the spread of the momenta among its electrons. The shape and size, as well as the trajectories of the individual electrons, may be altered. The resulting implications on the spatiotemporal resolution capabilities are discussed both for the $N$-electron pulse and for single-electron coherent packets introduced for microscopy without space-charge.

\section{Introduction}

In the investigation of complex systems ranging from biology to chemistry to materials science, it is beneficial to obtain structural information as a function of time. To achieve this goal, our laboratory has developed ${ }^{1}$ the ultrafast techniques of electron diffraction (UED), ${ }^{2}$ electron crystallography (UEC) $)^{3,4}$ and electron microscopy (UEM), ${ }^{5}$ for more details of the historical development see ref. 1 and references therein. In striving to identify the relevant degrees of freedom of the structural dynamics, the experimental tools for investigating fundamental physical, chemical and biological processes need to feature ever-improving spatiotemporal resolution. Using electrons as a probe, the resulting high sensitivity allows for the use of ultrashort pulsed beams with unprecedented time resolution, ranging from femtoseconds to picoseconds.

The temporal resolution is mainly determined by the longitudinal extent of the electron pulse, while the spatial resolution limits for both diffraction and imaging are determined by the same requirements that apply to continuous-wave beams. To establish and possibly improve upon the resolution limits of ultrafast electron diffraction and electron imaging instruments, detailed knowledge of the temporal evolution of electron pulses becomes crucial. Here, we present a comprehensive theoretical study of the relevant pulse broadening mechanisms and investigate their effect on the spatiotemporal resolution in electron diffraction, crystallography, and imaging. The geometrical factors caused by the velocity mismatch between the optical and electron pulses in a crossed beam arrangement are treated elsewhere ${ }^{6}$ and are not considered here. As discussed below, a tilted pulse geometry circumvents this problem in UEC.

Physical Biology Center for Ultrafast Science and Technology, Arthur Amos Noyes Laboratory of Chemical Physics, California Institute of Technology, Pasadena, CA 91125.E-mail:zewail@caltech.edu
The electron pulse trajectories are conceptually similar in all of the above-mentioned instruments. The ultrashort electron pulse is formed by illuminating a photocathode material with a pulsed laser beam, thereby generating free electrons by the photoelectric effect. The free electron packet is then accelerated through an electric field and subsequently shaped using an instrument specific combination of pinholes, electrostatic and magnetostatic lenses, as shown schematically in Fig. 1. However, the number of electrons per pulse varies considerably across our instruments: in gas-phase electron diffraction (UED), where the specimen's molecular density is low, the electron pulse contains up to $10^{6}$ electrons in order to maximize the signal intensity, while in electron crystallography (UEC), $\sim 10^{3}$ electrons per pulse results in sufficient scattering events (see Appendix A). Finally, in electron microscopy (UEM), single-electron pulses are employed at an increased repetition rate to build up an image or diffraction pattern in time.

Several factors come into play during the electron pulse trajectory and these factors determine the bunch shape at the specimen in the interaction region. First, the electrons are generated by a laser pulse, which itself has a temporal and spatial extent; the extent defines the initial shape of the electron packet. Second, the electrons are ejected from the photocathode with a range of momenta defined by the distribution in magnitude and direction. Third, at sufficiently high charge densities, pair-wise Coulomb repulsions between electrons become significant and such repulsions may alter the velocities of individual electrons. The latter effect is not operational in the single-electron mode.

Simulating electron packets realistically along the entire path is complicated due to the presence of instrument specific electron optical arrangements. While the electron microscope features a myriad of magnetostatic lenses, electrostatic lenses, deflection coils, stigmator coils and pinholes, the column of our UED or UEC instruments, containing just a single 


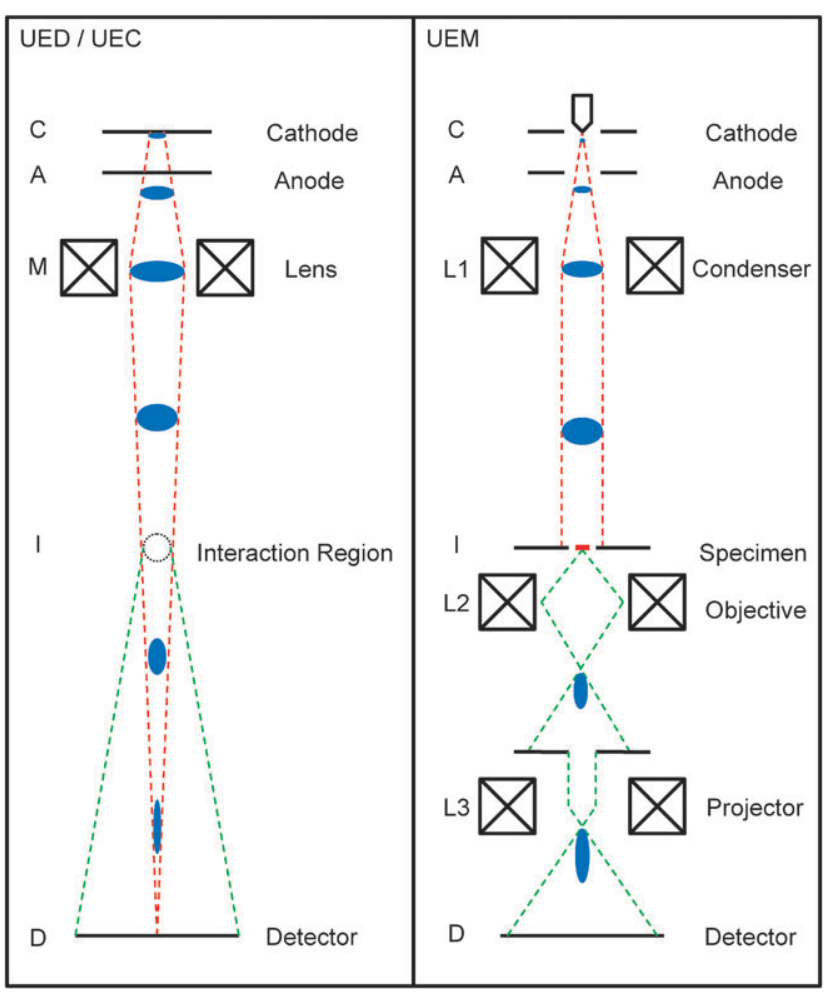

Fig. 1 Schematic of the optical column in UED/UEC and UEM. Electrons are generated by the photoelectric effect at the cathode (C) with the given profile, accelerated between a single electrode pair, radially focused by a solenoid coil (M). The electron pulse evolution is monitored, from the source until they reach the detector (D). In UEM, the pulses are shaped using lens systems (L1, L2, and L3), rather than simple solenoid coils.

magnetostatic lens, seems fairly simple by comparison. Theoretical studies to date ${ }^{7-11}$ have treated electron pulses for diffraction and imaging primarily in the absence of focusing fields, thereby relying on several assumptions to match experimental data, which could be violated in reality.

This paper is organized as follows: First we will consider the propagation of electron pulses in the absence of Coulomb repulsions to determine the magnitude of pulse broadening mechanisms, other than the space-charge effect. Second, the electron pulses in the presence of space-charge will be modeled with typical UED or UEC parameters using two approaches: a mean field model ${ }^{7,8,10}$ and an $N$-body Monte Carlo simulation. ${ }^{12}$ The mean field model will be expanded to allow for the incorporation of pulse shaping fields. It is shown that the Monte Carlo simulations give more accurate pulse shapes and, perhaps more importantly, deliver the complete phase space information of the ensemble of electrons. Together these two approaches provide a valuable illustration of the physical processes of electron-electron interactions within the pulse and their subsequent implications for electron-specimen interactions in diffraction and imaging. We conclude with a discussion about the beam coherence properties and apply these concepts to maximize the spatiotemporal capabilities of our newly-developed diffraction instrument, UED4. Comparisons with the experimental resolutions achieved are also made (see Fig. 2).

\section{Theory}

\section{A. Definition of width and length of packet}

Macroscopically, the electron packet is defined by its longitudinal and lateral spatial profiles and their evolution in time, as it travels from the photocathode to the interaction region and, finally, to the detector. Typically, these distributions can be assumed to be cylindrically uniform, ellipsoidal, Gaussian or their combinations. ${ }^{7}$ When comparing different shapes and distributions of electron packets, a natural measure for the spatial extent has to be defined. While the "maximum extent" would be an obvious choice for a uniform distribution, the full-width-half-maximum (FWHM) is often used for a Gaussian distribution and for experimental measurements; in few cases, the width at $10 \%$ height and/or the standard deviation, $\sigma$, is also used. For the uniform cylindrical and/or ellipsoidal distribution, the measurement of the diameter often implies a projection of the $2 \mathrm{D} / 3 \mathrm{D}$ density onto the respective axis, resulting usually in a Gaussian-type function. Furthermore, real electron packets do not necessarily maintain their initial shapes, during expansion, with the noted exception of ellipsoidal packets. ${ }^{13}$

For quantitative comparison of the results obtained from the mean field theory, the numerical simulation, and experiment, we choose the standard deviation as the universal metric of size. Two packets are deemed "equivalent" when they have the same standard deviations regardless of the shape of the distribution. Only if the actual distribution shape is known or assumed, then the standard deviation can be related to more common measures of the spatial extent of the pulse, such as the FWHM (see Appendix B for conversions). In the mean field theory, for example, in order to simulate a $110 \mathrm{fs}$ FWHM Gaussian pulse ( $\sigma=47 \mathrm{fs}$ ), we would choose a uniform length of $162 \mathrm{fs}(=\sqrt{12} \times 47 \mathrm{fs})$. Alternatively, Miller and coworkers, ${ }^{11}$ instead of converting the measure of size, scaled the number of electrons in the packets to match the results of the mean field theory and their $N$-body simulation, since the FWHM of a Gaussian distribution contains only $76 \%$ of the total electrons.

\section{B. Initial energy spread}

In the photoelectric process, free electrons are generated with a distribution of kinetic energies. In order to evaluate the magnitude of the pulse broadening it is instructive to treat the space-charge effect separately from the broadening due to the initial energy spread. To this end, the relativistic equation of motion has been solved for a single electron having an uncertainty in its momentum. Longitudinal broadening, $\Delta t_{\mathrm{KE}}$, will result from an initial spread in the electron kinetic energy, $\Delta E_{\mathrm{i}}$, or the corresponding momentum spread, $\Delta p_{\mathrm{i}}=\sqrt{\frac{m_{0}}{2 E_{\mathrm{i}}}} \Delta E_{\mathrm{i}}$, which occurs during the electron photoemission event (see Appendix C):

$$
\Delta t_{\mathrm{KE}}=\left|-\frac{d}{e V}\left(1-\frac{v_{\mathrm{i}}}{v_{\mathrm{f}}}\right)-\frac{l}{m_{0} \gamma_{\mathrm{f}}^{3}} \frac{v_{\mathrm{f}}}{v_{\mathrm{f}}^{3}}\right| \Delta p_{\mathrm{i}}
$$

where $e$ is the electron charge, $m_{0}$ is the electron rest mass, $d$ is the acceleration gap between cathode and anode, $l$ is the distance of field-free propagation, $V$ is the acceleration 

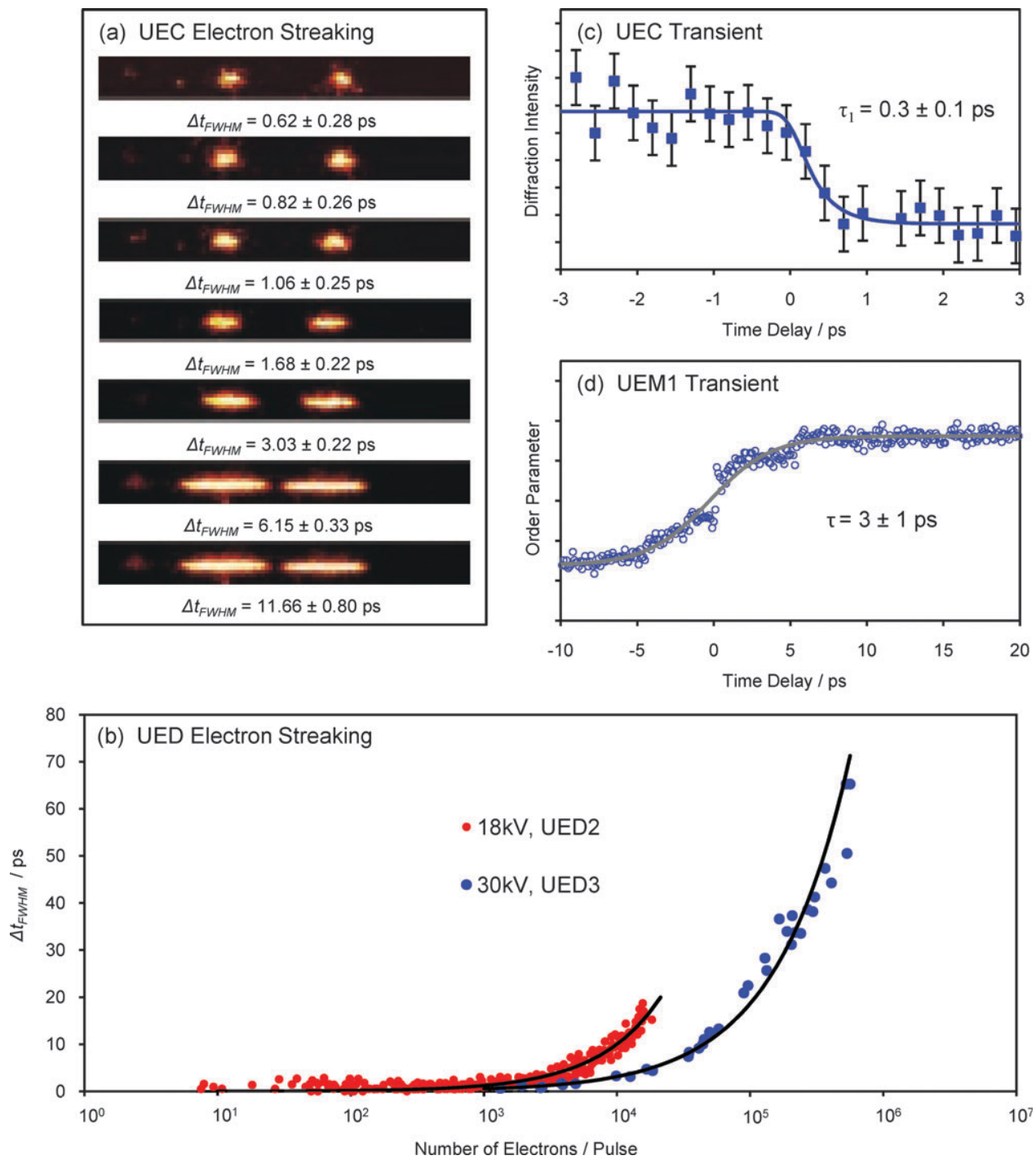

Fig. 2 Measured resolutions for UEC and UED and experimental transients obtained by UEC and UEM1. (a) Streaked electron pulses on the CCD (charge-coupled device) detector together with the calculated pulse lengths. (b) Measured electron pulse widths as a function of the number of electrons. The blue curve (UED3) shows more than an order-of-magnitude improvement in the electron gun performance in comparison to the red curve (UED2). The number of electrons for the UED3 measurement in ref. 20 was given as density (electrons $\mathrm{mm}^{-2}$ ). For the data shown here, the original streak images have been reanalyzed and they are now given in terms of the absolute number of electrons. The lines are drawn as best fits, but the theoretical curves are given in Fig. 6. (c) Ultrafast dynamics of structural phase transition in vanadium dioxide. Intensity change of the (606) Bragg spot with time. A decay with a time constant $\tau_{1}$ of $307 \mathrm{fs}$ was reported in ref. 31 . Here the data was deconvoluted (electron pulse width of $344 \mathrm{fs}$ ) and we obtained $\tau_{1}=0.3 \pm 0.1 \mathrm{ps}$. (d) Temporal evolution of the structural order parameter. The order parameter is defined as the integrated intensity of the diffraction peak for different temporal frames. Adopted from ref. 3, 20, 31, and 38.

voltage, $v_{\mathrm{i}}$ and $v_{\mathrm{f}}$ are the mean initial and final electron velocities, $\gamma_{\mathrm{f}}$ is the relativistic Lorentz factor (see Appendix C) at the velocity $v_{\mathrm{f}}$.

The first term represents the broadening of the electron pulse in the acceleration gap, the dominant contribution, while the second term represents broadening in the field-free drift region. We note that the second term corresponds to a packet simply spreading by $l /\left(v_{\mathrm{f}} \pm \Delta v_{\mathrm{f}}\right)$ in the drift region. Since $v_{\mathrm{i}} \ll v_{\mathrm{f}}$, the expression can be approximated to yield

$$
\Delta t_{\mathrm{KE}} \approx \frac{d}{e V} \Delta p_{\mathrm{i}}=\frac{d}{e V} \sqrt{\frac{m_{0}}{2}} \frac{\Delta E_{\mathrm{i}}}{\sqrt{E_{\mathrm{i}}}}
$$

giving a result equivalent to that of eqn (4) in ref. 14. It should be noted that, under this approximation, the temporal spread is solely determined by the energy spread, $\Delta E_{\mathrm{i}}$, relative to the square root of the mean energy, $\sqrt{E_{\mathrm{i}}}$, and the potential $V$ across the distance $d$.

\section{Charge density effect}

1. Mean field theory (MF). In the mean field theory, which has been widely used in the literature, ${ }^{7-11}$ the electron pulse is most commonly approximated as a cylindrical slab of radius $R$ and length $L$. Due to the space-charge effect, this slab of continuous charge density extends both in the longitudinal 
and transverse direction, while always maintaining the shape of a cylinder. The evolution of the spatial pulse length, $L$, due to space-charge repulsion is given by (see Appendix D for derivation):

$$
\frac{1}{2} \frac{\mathrm{d}^{2} L}{\mathrm{~d} t^{2}}=a_{\mathrm{X}}=\frac{N e^{2}}{2 m_{0} \varepsilon_{0} \pi R^{2}} \frac{2}{1+\left(\frac{L}{R}\right)+\sqrt{1+\left(\frac{L}{R}\right)^{2}}}
$$

where $a_{\mathrm{X}}$ is the axial acceleration, $N$ is the number of electrons in the pulse, and $\varepsilon_{0}$ is the vacuum permittivity. The time evolution of the radius, $R$, of a freely expanding, thin disk $(R \gg L)$ is approximated by ${ }^{8}$

$$
\frac{\mathrm{d}^{2} R}{\mathrm{~d} t^{2}}=a_{\mathrm{R}} \approx \frac{N e^{2}}{m_{0} \varepsilon_{0} \pi R^{2}}
$$

where $a_{\mathrm{R}}$ is the radial acceleration. The temporal spread, $\Delta t_{\mathrm{SC}}$, is then evaluated from the length of packet and its axial velocity,

$$
\Delta t_{\mathrm{SC}}=\frac{L}{v_{f}}
$$

Here, the capital letters, $\Delta$ and $L$, are used to emphasize that the pulse duration corresponds to the maximum extent of the uniform distribution. The initial condition for $L$ is chosen to match the excitation laser duration, as previously described.

The 1-dimensional mean field model (MF1D) utilizes eqn (3) only with a constant radius, while the coupled eqn (3) and (4) are solved simultaneously to give a 2-dimensional result (MF2D). The advantage of the mean field model is that the equations of motion for the pulse can be rapidly integrated for a variety of initial conditions and give a readily estimate of the pulse size as a function of time. To date, the mean field model has been used primarily to model freely drifting electron bunches. While several additions to the theory have been made, e.g. to model the electron bunch inside an electrostatic acceleration field, ${ }^{9,15}$ no attempts have been made to model the effect of a magnetic lens on the electron pulse shape. Since these fields are present in all electron diffraction and imaging instruments, this extension to the mean field theory has to be made to validate its predictive power.

2. Mean field theory including lens system. To implement the acceleration field inside the electron gun, the mean field theory can be extended by treating the positions at the front and the rear end of electron packet separately and explicitly, as $F(t)$ and $B(t))^{9,15}$ The maximum longitudinal extent is then given by $L(t)=F(t)-B(t)$. Approximating the focusing lens as a radial deceleration element, the equation of motion becomes

$$
\begin{aligned}
& \frac{\mathrm{d}}{\mathrm{d} t}\left(\gamma_{\mathrm{F}} \frac{\mathrm{d} F(t)}{\mathrm{d} t}\right)=a_{\mathrm{A}}+a_{\mathrm{X}}\left(t \geq t_{\text {birth,front }}\right) \\
& \frac{\mathrm{d}}{\mathrm{d} t}\left(\gamma_{\mathrm{B}} \frac{\mathrm{d} B(t)}{\mathrm{d} t}\right)=a_{\mathrm{A}}-a_{\mathrm{X}}\left(t \geq t_{\text {birth,rear }}\right) \\
& \frac{\mathrm{d}}{\mathrm{d} t}\left(\gamma_{\mathrm{C}} \frac{\mathrm{d} R(t)}{\mathrm{d} t}\right)=a_{\mathrm{R}}-a_{\mathrm{M}}
\end{aligned}
$$

where $\gamma_{i}$ is the relativistic Lorentz factor for front, rear, and center, respectively, $a_{\mathrm{X}}$ and $a_{\mathrm{R}}$ are the axial and the radial accelerations as in eqn (3) and (4), $a_{\mathrm{A}}$ is the electrostatic acceleration in the electron source, and $a_{\mathrm{M}}$ is the deceleration term due to the focusing lens, expressed as a Gaussian function to simulate the finite thickness of a magnetic lens. It should mentioned that the axial forces are present only after the births of the front and the rear and that the number of electron is now a function of time, which is zero before the birth of the front, $N$ after the birth of the rear, and linearly increasing in between to mimic the generation of the photoelectrons.

3. Monte Carlo simulation (MC). In contrast to the mean field model, which treats the electron pulse as a continuous charge distribution, a $N$-body Monte Carlo simulation treats the electron pulse as an ensemble of $N$ randomly generated, discrete particles. ${ }^{12}$ To this end, we have developed our own electron bunch propagation code, in which each particle in the bunch moves under the influence of three distinct forces: (1) the electrostatic force of acceleration, (2) the magnetostatic force of the focusing lens, and (3) the Coulomb force for each of the $N(N-1) / 2$ pair-wise interactions within the bunch. The magnetic lens can be simulated by either (a) a sum of current loops, (b) the finite-sized coil approximation or (c) by importing an externally simulated field. Further elements such as the ponderomotive force, gravitation, the earth magnetic field, stigmators coils, and time-dependent streak plates can be straightforwardly implemented, if desired.

Using this physically more realistic model, it is possible to propagate the electron pulse over its entire lifetime, i.e. from the birth of the individual photoelectrons at the photocathode surface to their arrival at the detector. The nascent photoelectrons are randomly generated at the photocathode with a Gaussian temporal and uniform spatial distributions to account for the fact that the extraction pinhole in the anode acts as a spatial filter producing an initially well defined lateral profile. The direction of the initial electron momentum vectors are given by a $\cos ^{2} \theta$ distribution and the initial momentum distribution is chosen to be uniform from zero to a high energy cutoff corresponding to $h \nu-W$, where $W$ is the effective material-specific work function in the presence of a DC electric field. ${ }^{16}$ The treatment of the individual electron trajectories is entirely relativistic and should reproduce the true pulse trajectory, provided that the initial conditions are chosen accurately and the Monte Carlo sampling is fine-grained enough that the results converge. The drawback of this method is its high demand of computational time, which increases in proportion to the number of pair-wise interactions calculated at each time step. To ease computational demand, we treated the pulse as an ensemble of representative particles of appropriately scaled charge and mass in order to model pulses containing more than 1000 electrons in a reasonable amount of time. We found that this level of Monte Carlo sampling was sufficient to achieve convergence to within $10 \%$.

\section{Results and discussion}

\section{A. Temporal resolution}

1. Initial energy spread. In order to avoid pulse broadening due to Coulomb repulsion and achieve ultrafast temporal 
resolution, the ultrafast electron microscope has been designed to operate in the zero-current limit, meaning that the column contains one or a few electrons at a time. For an instrument of this type, the ultimate resolution is mainly determined by the excitation laser pulse length and by the initial kinetic energy spread of the photoelectrons. The pulse broadening in the absence of space-charge is calculated from $\Delta t=\sqrt{\Delta t_{h \nu}^{2}+\Delta t_{\mathrm{KE}}^{2}}$ with $\Delta t_{\mathrm{KE}}$ given by eqn (1) or eqn (2). Using instrumental parameters, the results are shown in Fig. 3 for $\Delta t_{\text {FWHM }} v s . \Delta E_{\mathrm{i}}$. We invoke, for simplicity, a uniform photoelectron distribution (see ref. 17) centered at $E_{\mathrm{i}}$ and the width increase given by $\Delta E_{\mathrm{i}}$ $=2 E_{\mathrm{i}}$. The exact solution (solid lines, eqn (1)) represents the broadening of the pulses during their flight to the interaction region, while the approximate solution (dashed lines, eqn (2)) represents the broadening in the acceleration region. Since eqn (1) and (2) give very similar results, the broadening in the field-free drift region is relatively insignificant.

As evident from eqn (2), it would be desirable to produce photoelectrons with a narrow energy distribution, but a large mean kinetic energy to reduce the magnitude of the factor $\Delta E_{\mathrm{i}} / \sqrt{E_{\mathrm{i}}}$. Experimentally, however, the distribution of photoelectrons always extends to the limit of zero kinetic energy, because electrons below the Fermi level may be photo-emitted as well. ${ }^{17}$ To achieve the narrowest possible energy distribu- tion, the photocathode needs to be operated at a low temperature and the photon energy needs to be matched to the work function of the metal. ${ }^{18}$ This approach is taken in our instruments of UED and UEC, resulting in an estimated kinetic energy spread of $<0.3 \mathrm{eV}$; in ref. 18 , the experimental $\Delta E_{\mathrm{i}}$ under similar conditions (for a gold photocathode) was measured to be $0.1 \mathrm{eV}$.

We consider the electron pulses produced in UED4 and UEC first. The acceleration gap in these guns is kept as small as possible to maximize the extraction field. Under the influence of a field strength of $20 \mathrm{MV} \mathrm{m}^{-1}$ and $10 \mathrm{MV} \mathrm{m}^{-1}$ for UED4 and UEC, respectively, the electron pulses are barely elongated by the presence of an initial kinetic energy spread. In contrast, the broadening is more pronounced in UEM1 due to the smaller acceleration field strength of $4.8 \mathrm{MV} \mathrm{m}^{-1}$. The UEM1 column, originally designed to operate in continuouswave mode, features an acceleration gap one order of magnitude larger than the gap found in the home-built guns of UEC and UED. Nonetheless, even at $\Delta E_{\mathrm{i}}=0.3 \mathrm{eV}$, the pulse length is only $300 \mathrm{fs}$.

Since UEM operates in the absence of space-charge, the photoelectric energy spread presents the main contribution to the electron pulse broadening. If necessary, the electron pulse duration could be reduced to the excitation laser pulse length by an appropriately designed extraction module. The

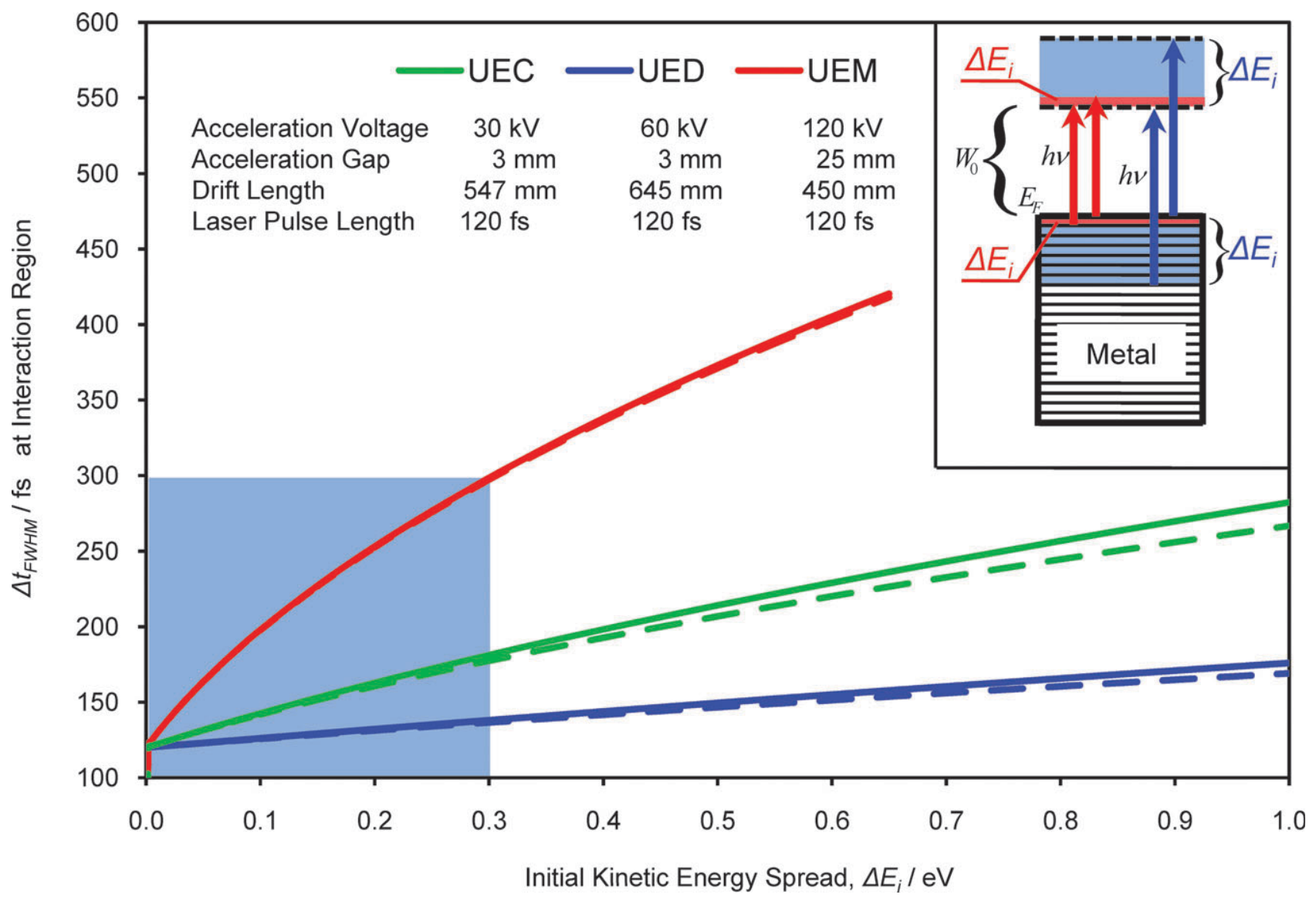

Fig. 3 Temporal broadening of the electron pulse due excess energy of the electrons above the photocathode work function for the UED4 (blue), UEC (green) and UEM1 (red) instruments. The pulse length at the specimen interaction region is computed in the absence of space-charge using the instruments specific parameters. In the inset, the red arrow indicates the excitation from near the Fermi surface $\left(E_{\mathrm{F}}\right)$ to just above the vacuum, while the blue arrow is for excitation which carries an excess of photon energy. 
consequence of the energy distribution, $\Delta E_{\mathrm{i}}$, is in another aspect of diffraction and imaging, namely the longitudinal coherence, which will be discussed below.

2. Space-charge effect. Before we evaluate by how much the pulse durations shown in Fig. 3 will be altered by the space-charge effect, we need to validate the different methods of simulating the electron packet. Fig. 4 shows the size (standard deviation) evolution of an electron bunch under the exclusive influence of Coulomb repulsions $\left(\Delta E_{\mathrm{i}}=0\right)$, calculated using the mean field theory (i) in the longitudinal direction only (MF1D), (ii) in two dimensions with the implementation of a focusing element (MF2D), (iii) in two dimensions with the implementation of a focusing element and the acceleration field (MF2DA), and, ultimately, the Monte Carlo (MC) simulation. We note that $\sigma_{\text {temporal }}$ in $\mathrm{mm}$ is the longitudinal extent of the space-charge limited electron packet and, knowing the speed (post acceleration), can be expressed in the time domain using eqn (5). The initial conditions were chosen as follows: 10000 electrons per pulse, $30 \mathrm{kV}$ acceleration voltage, Gaussian FWHM (110 fs) or the equivalent uniform profiles in time, uniform profile in the lateral direction $(r=100 \mu \mathrm{m})$. In each case, the current of the magnetic lens was optimized, such that the electron beam would have the smallest beam waist as it hits the detector.
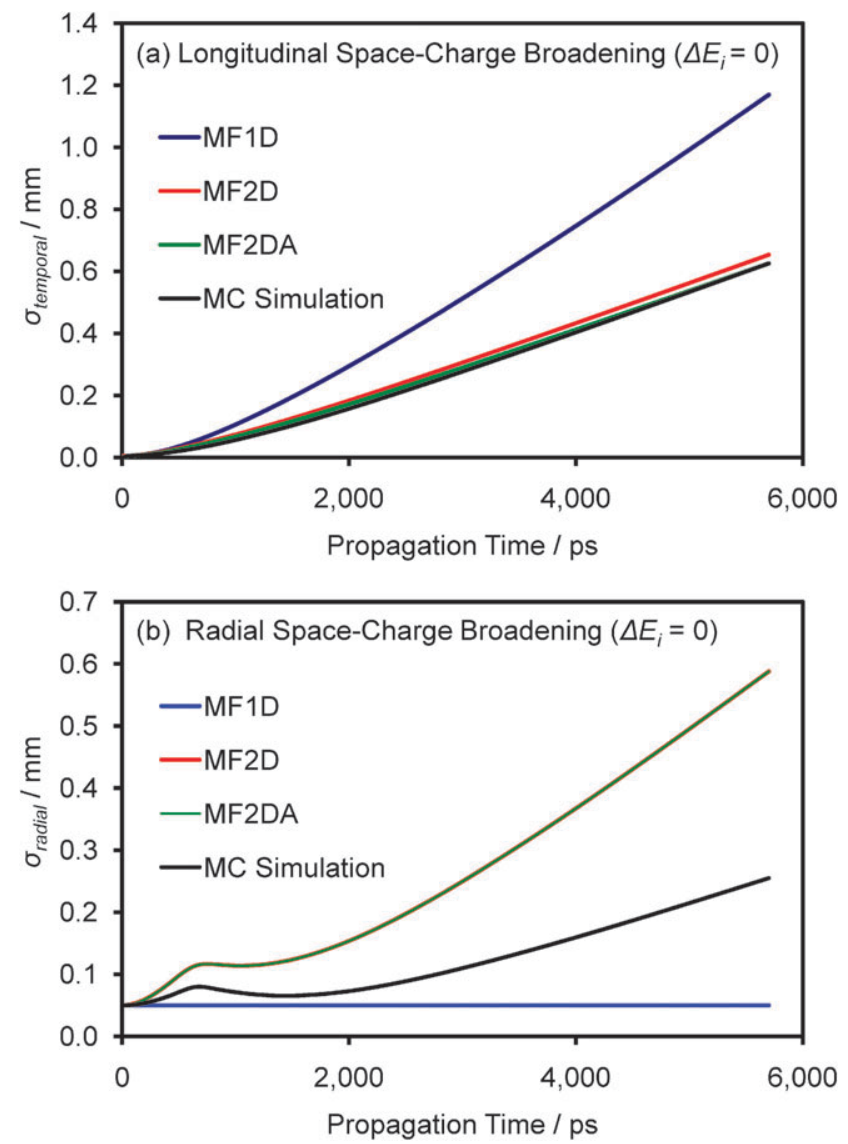

Fig. 4 Comparison of the length (a) and the radius (b) of the electron pulse predicted by the mean field theories and the $\mathrm{N}$-body Monte Carlo simulation in the absence of an initial kinetic energy spread.
Not surprisingly, the MF1D approximation with a fixed radius clearly overestimates the longitudinal spreading of the pulse, while the other methods give a good agreement. The radial acceleration used in the mean field models (eqn (4)) is only valid for infinitely thin disks and, therefore, overestimates the spreading in the lateral direction as the pulse elongates, such that even after optimizing the magnetic lens, the radius remains too large. In spite of this large error, the longitudinal spread is only affected slightly, at least at this particular charge density. We can conclude that the mean field model may give a reasonable estimate of the pulse duration for a space-charge limited beam for a choice of arbitrary initial conditions. However, the radial expansion is not accurately reproduced by the current model and would require modification of eqn (4).

The extent of the space-charge-induced broadening is mainly determined by the magnitude of the Coulomb repulsions, as well as the time scale of this interaction. By employing higher acceleration voltages, the effective propagation time of the electron pulse can be reduced, since the electrons arrive at the interaction region in a shorter time and the space-charge induced broadening has less time to act. We used the mean field model to estimate the pulse length in our instruments as a function of propagation distance. The extraction voltages are 30, 60 and $120 \mathrm{kV}$ for UEC, UED4, and UEM1, respectively. The effect of the acceleration voltage on the temporal duration (Gaussian FWHM) of the pulses as a function of the propagation distance is shown in Fig. 5. It is clear that UEM1 in single-electron pulsed mode is not limited by the space-charge effect, while the pulses in UED4 and UEC are broadened to several ps once they arrive at the interaction region. Alternatively, the time of the space-charge induced broadening could also be reduced by placing the interaction region very close to the electron source. ${ }^{19}$

To isolate the role of the initial charge density, we calculated the temporal extent of the electron pulses under identical acceleration conditions. The initial conditions were kept identical to the conditions used for the comparisons of the models in Fig. 4, while the number of electrons was increased by an

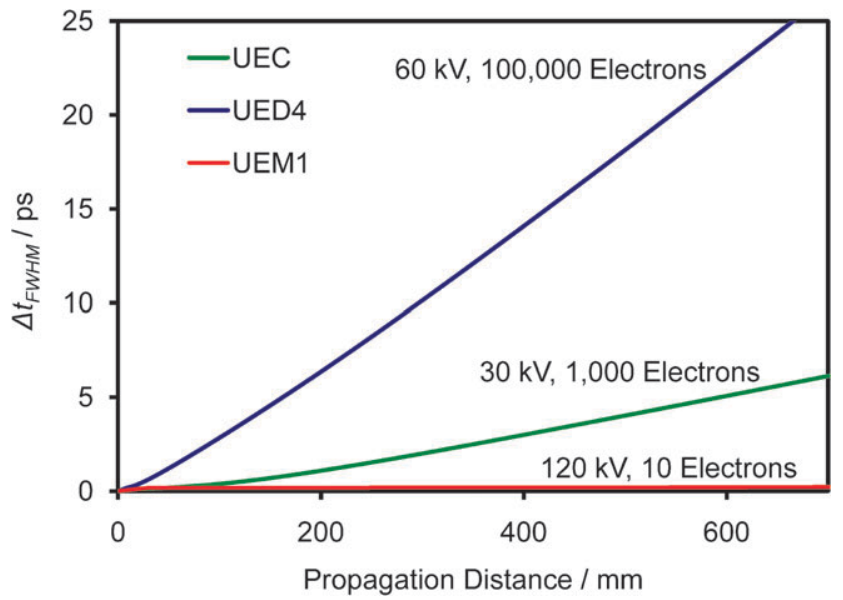

Fig. 5 Comparison of temporal broadening due to the space-charge effect $\left(\Delta E_{\mathrm{i}}=0\right)$ as a function of the propagation distance in UEC (green line), UED4 (blue line), and UEM1 (red line) using MF2DA. 


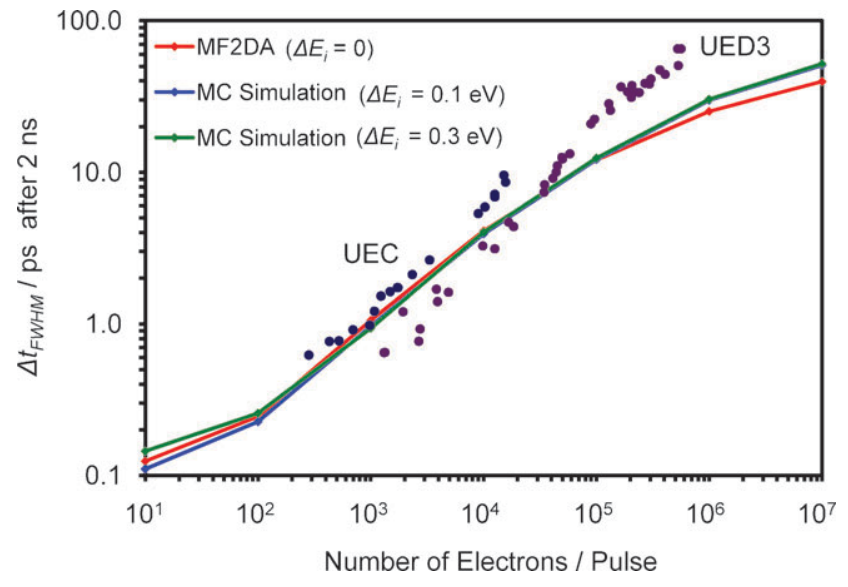

Fig. 6 Comparison of total temporal broadening due to the space-charge effect after 2 ns of propagation using MF2DA (blue line), $\mathrm{MC} 0.1 \mathrm{eV}$ (red line), and $\mathrm{MC} 0.3 \mathrm{eV}$ (green line). Available experimental data are given for UEC (blue dots), and UED3 (red dots); see Fig. 2 (UEC and UED3, $30 \mathrm{kV}$ ).

order of magnitude at a time. Fig. 6 shows the theoretical (MF2DA and MC) pulse duration after $2 \mathrm{~ns}$ of propagation time together with the experimental measurement for validation. $^{20}$ Both models reproduce the pulse broadening reasonably well in the region where experimental data is available. Using the MC simulation, two curves were calculated for different initial kinetic energy spreads corresponding to 0.1 and $0.3 \mathrm{eV}$. There is a slight difference between these two calculations in the low electron density regime, where the space-charge effect does not play a dominant role. However, the curves quickly begin to overlap each other as the electron density grows and for a pulse containing as few as 1000 electrons, the space-charge-induced broadening already masks any contribution of the initial kinetic energy spread after 2 ns of propagation. The mean field model can reproduce the results from the MC simulation quite accurately, but a more pronounced deviation occurs at higher charge densities, since the error in eqn (4) is exacerbated in this regime. Again, the temporal resolution capability of the pulsed electron probe can be successfully estimated with the mean field model; however, the spatial resolution capability remains uncertain.

\section{B. Spatial resolution}

1. Coherence. The evaluation of the spatial resolution of the pulsed electron probe requires a detailed discussion of the coherence of the electron packet. Coherence is the degree of a phase relationship, which can give rise to interference. In light optics, an aperture is often employed to generate a pseudo point source. For a single illuminated object, the analogue of the double-slit experiment, the coherence length is defined as the maximum length beyond which the interference fringe is attenuated. Below, we will consider the effect of many, spatially separated objects (interaction region). If the aperture is small and the distance to the object is far, then the coherence length is defined as $r_{\mathrm{c}}=\lambda /(2 \pi \alpha)$, where $\alpha$ is the half-angle subtended by the aperture. In such a case, the object is illuminated by spherical waves emanating from every point of the aperture. It should be mentioned that the criterion $r_{\mathrm{c}}=$ $\lambda /(2 \pi \alpha)$ corresponds to only a $12 \%$ reduction of the perfectly coherent visibility. ${ }^{21}$ This definition holds true, only as long as $\alpha$ is smaller than the photon's intrinsic divergence, $\delta \alpha$, which can be estimated using the uncertainty principle:

$$
\delta \alpha \approx \frac{\Delta p_{r}}{p_{z}} \geq \frac{\hbar}{2 \Delta x} \frac{\lambda}{h}=\frac{\lambda}{4 \pi \Delta x}
$$

where $p_{r}$ and $p_{z}$ are the photon momenta in the radial and longitudinal direction, respectively, and $\Delta x$ is the aperture dimension. However, when $\alpha$ is bigger than $\delta \alpha$, then the object is illuminated only by an area within the angle $\delta \alpha$ and the contribution from the rest of the source can be neglected. In such a case, the coherence length should be defined as $r_{\mathrm{c}}=\lambda /(2 \pi \delta \alpha)$.

In contrast to photons, free electrons are generated with an initial momentum spread, which determines the intrinsic divergence $\delta \alpha$ for each electron, since the contribution originating from the uncertainty principle term is negligible due to the small de Broglie wavelength. Using an acceleration voltage of $60 \mathrm{kV}$ and assuming $\Delta E_{\mathrm{i}} \approx 2 E_{\mathrm{i}} \approx 0.3 \mathrm{eV}$, the value of

$$
\delta \alpha=\frac{\Delta p_{r}}{p_{\mathrm{f}}} \leq \frac{\Delta p_{\mathrm{i}}}{p_{\mathrm{f}}} \approx \sqrt{\frac{\frac{m_{0}}{2 E_{\mathrm{i}}}\left(\Delta E_{\mathrm{i}}\right)^{2}}{2 m_{0} E_{\mathrm{f}}}}=\sqrt{\frac{\left(\Delta E_{\mathrm{i}}\right)^{2}}{4 E_{\mathrm{i}} E_{\mathrm{f}}}}
$$

becomes $1.6 \mathrm{mrad}$, which is an upper limit value. (The presence of a $\delta \alpha$ ultimately gives rise to a non-zero transverse beam emittance. ${ }^{22}$ )

The coherence length defined above only applies for a single scattering object. Blurring of the interference fringes needs to be taken into account in the case of scattering from objects with spatial extent as in molecules or in the condensed phase. In this context, it is more appropriate to derive the coherence length in terms of interference fringe blurring. During the elastic scattering process, the magnitude of the momentum transfer is given by

$$
s=\left|\vec{k}-\vec{k}_{0}\right|=\frac{4 \pi}{\lambda} \sin \left(\frac{\theta}{2}\right)
$$

where $\overrightarrow{k_{0}}$ and $\vec{k}$ are incident and scattered wave vectors of the electron, $\lambda$ is the electron's de Broglie wavelength, and $\theta$ is the scattering angle. When the ensemble of electrons and the interaction volume have a finite size, each electron has an uncertainty in its position, when it scatters. The error in the observable momentum transfer at a given radial position $s$ on the observation plane can be evaluated by

$$
\begin{aligned}
& \delta s= \\
& \sqrt{\left(\frac{\partial s}{\partial \theta}\right)^{2} \delta \theta^{2}+\left(\frac{\partial s}{\partial \lambda}\right)^{2} \delta \lambda^{2}+\left(\frac{\partial s}{\partial x}\right)^{2} \delta x^{2}+\left(\frac{\partial s}{\partial y}\right)^{2} \delta y^{2}+\left(\frac{\partial s}{\partial z}\right)^{2} \delta z^{2}+\left(\frac{\partial s}{\partial b}\right)^{2} \delta b^{2}}
\end{aligned}
$$

The $\delta \theta$ term represents the uncertainty in the scattering angle, originating from the incident angular spread at each position in the interaction volume (lateral coherence length), the $\delta \lambda$ term represents the longitudinal energy spread (longitudinal coherence length) and the $\delta x, \delta y$ and $\delta z$ terms are reflective of the 3-dimensional size of the interaction region and the electron bunch. The $\delta b$ term is an uncertainty in measuring the momentum transfer due to the electron detection process, e.g. through blurring and binning the image (signal converter, 
amplifier, and digitizer). In case of gas-phase electron diffraction, each distinct internuclear distance produces sinusoidal interference fringes, ${ }^{23}$ while in crystallography, repeated longrange order of scattering centers produces a series of Bragg spots with a corresponding frequency in spacing. ${ }^{24}$ The uncertainty in $s$ will reduce the visibility of the interference fringes or the Bragg spots, such that they become unobservable and indistinguishable (see Appendix E). The instrumental coherence length can then be defined as

$$
R_{\mathrm{c}}=\frac{1}{\delta s} \equiv \frac{1}{2 \sigma_{s}}
$$

We note that this definition reduces to $r_{\mathrm{c}}=\lambda /(2 \pi \delta \alpha)$, the lateral coherence length, only in the single scattering center limit and assuming the detection process is perfect and $\delta \lambda$ is negligible. Additionally, from discrete Fourier transformation theory, it is known that the maximum resolvable distance after collecting discrete data in the frequency domain, is given by

$$
r_{\max }=\frac{1}{\Delta s}=\frac{1}{s_{i+1}-s_{i}}
$$

Therefore, these values give an estimate of the maximum distance that can be decisively resolved in an electron diffraction experiment.

In the high resolution imaging mode of the transmission electron microscope, the coherence of the electron ensemble is exploited to form contrast in the image. Conceptually, the same limitations as stated above apply for the instrument's spatial resolution. Since each electron interferes only with itself, the image is composed of the independent superposition of single electron interferences and image blurring occurs in the presence of an uncertainty in the electrons' trajectory and their wavelength.

However, in the more complicated microscope column additional resolution limiting factors have to be considered. In the wave-optical theory of imaging, ${ }^{25}$ contrast in the image is formed as dictated by the phase contrast transfer function (CTF), which is a function not only of the properties of the electron beam (incidence angle spread and wavelength spread) but also of instrumental parameters such as the spherical and chromatic lens aberrations, as well as aperture sizes and the particular defocus setting. Most commonly used is the Scherzer defocus, which, in combination with an appropriate aperture size, maximizes the contrast and allows for easily interpretable images. The Scherzer resolution can be improved upon by exit wave reconstruction techniques using images collected at different defocus settings, but the ultimate information limit of the instrument remains limited by the beam properties and the quality of the electron optics. Since UEM operates outside of the space-charge limit, the spatial resolution achievable is the same as is obtained in continuous-wave mode and we will not comment on it further.

2. Optimal diffraction geometry. In conventional diffraction physics, ${ }^{26}$ it has been established that $\delta s$ is minimized, when, for each electron, the incident wave vector, $\vec{k}_{0}$, is coincident with a line from the electron's position in interaction volume to the center of detector. As is shown schematically in Fig. 7, the most extensive blurring occurs, if the beam arrives at the
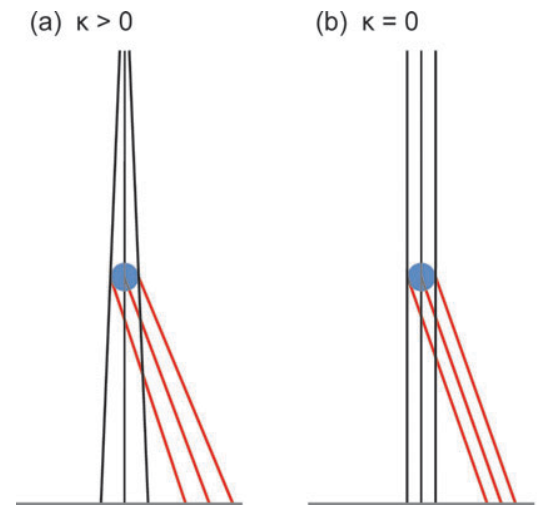

(c) $\mathrm{K}<0$

Fig. 7 Effect of the beam geometry on interference blurring for a finite sized beam using diverging (a), collimated (b), or converging (c) electron trajectories.

interaction region on a diverging trajectory, i.e. the particles' radial divergence angle, $\kappa=\tan ^{-1}\left(p_{r} / p_{z}\right)>0$. On the other hand, if the electrons are perfectly collimated $(\kappa=0)$, then the blurring on the detector is identical to the size of the electron beam waist. The optimal resolution is achieved, if the electrons are focused to a point on the center of the detector and impinge on the interaction region on an ideally-focused converging $(\kappa<0)$ trajectory. It follows that for a given camera length, the ideal radial convergence angle, $\kappa$, in the interaction region is given by an approximately linear function of the off-axis distance. Using an interaction volume with dimensions $\delta x=\delta y=\delta z=300 \mu \mathrm{m}$, an intrinsic electron divergence $\delta \theta=0.5 \mathrm{mrad}$, a wavelength spread $\delta \lambda$ corresponding to a kinetic energy spread of $0.3 \mathrm{eV}$, and a detector blurring $\delta b=100 \mu \mathrm{m}$, a coherence length of $\sim 12 \AA$ is achieved in the converging beam configuration, while the collimated beam and the diverging beam only give $\sim 4$ and $\sim 3 \AA$, respectively. The $\delta \lambda$ term in eqn (10) is much smaller compared to the remaining terms, such that the blurring of the interference fringes is not affected by longitudinal coherence.

3. Focusing behavior. In the absence of Coulomb repulsion, $\delta \theta$, which originated from the intrinsic divergence, $\delta \alpha$, will lead to a finite spot size on the detector. Therefore, the ability to focus the beam to a small point on the detector can be a direct measure of the instrumental coherence length, because $\delta s$ is dominated by $\delta \theta$ after all other terms have been minimized.

For high energy electrons, the de Broglie wavelength is much smaller than the source dimension and we can rather treat each electron in the bunch as a classical particle and its trajectory as a ray, which is influenced by external forces, if any. However, due to the presence of $\delta \alpha$, the focusing behavior of the electron beam becomes less than ideal, as shown schematically in Fig. 8. Assuming that the electrons are generated in a source of radius $R_{0}$, having an overall beam divergence $\Omega_{0}$, if any, and further assuming that each emitting point in the source inherently diverges with an angle $\delta \alpha$ after acceleration, then these two components are focused at different positions. The focal distances, $B$ and $b$, produced by a lens of a focal length, $f$, for the $\Omega_{0}$ and $\delta \alpha$ components, respectively, 


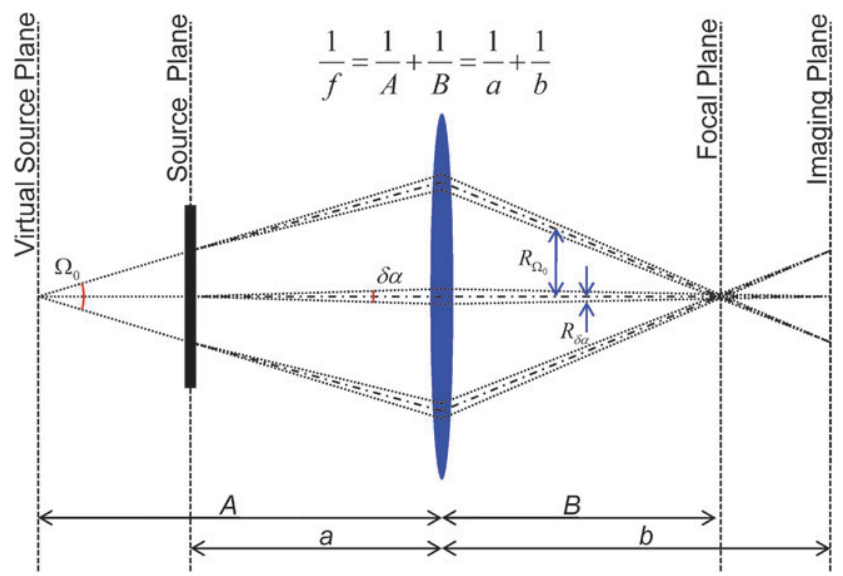

Fig. 8 Radial focusing behavior of a finite-sized beam in the absence of space-charge.

are given by the lens equation:

$$
\begin{aligned}
& \frac{1}{f}=\frac{1}{A}+\frac{1}{B} \text { for } \Omega_{0} \\
& \frac{1}{f}=\frac{1}{a}+\frac{1}{b} \text { for } \delta \alpha
\end{aligned}
$$

The $\delta \alpha$ components are perfectly focused at the imaging plane $b$, where a magnified image of the source can be formed, while the smallest overall beam waist is obtained near the focal plane $B$. If the radius due to each component ( $\delta \alpha$ and $\Omega_{0}$ ) can be determined independently from the two different focal distances, then the resulting beam size along the beam path may be computed by convoluting the radii of these two components, i.e.

$$
R(x)=\sqrt{R_{\Omega_{0}}(x)^{2}+r_{\delta \alpha}(x)^{2}}
$$

where

$$
R_{\Omega_{0}}(x)=\left|R_{0} \frac{A}{A-a} \frac{B-x}{B}\right|=\left|R_{0} \frac{A}{A-a}\left(1+\frac{x}{A}-\frac{x}{f}\right)\right|
$$

and

$$
\begin{aligned}
r_{\delta \alpha}(x) & =\left|a \tan \left(\frac{\delta \alpha}{2}\right) \frac{b-x}{b}\right| \\
& =\left|a \tan \left(\frac{\delta \alpha}{2}\right)\left(1+\frac{x}{a}-\frac{x}{f}\right)\right|
\end{aligned}
$$

and

$$
A=a+R_{0} \cot \left(\frac{\Omega_{0}}{2}\right)
$$

The optimal focal length of the lens can then be determined by minimizing $R$ with respect to $f$. Fig. 9a shows a result of eqn (14), (15), and (16) using typical UED parameters. It can be readily seen that, even though the $\Omega_{0}$ component is focused, the minimized spot size is finite and effectively determined by the diverging $\delta \alpha$ component. Sufficiently small radii can be obtained either by (i) making $R_{0}$ very small (point source), or (ii) by making $\Omega_{0}$ large. Both approaches essentially bring the virtual source position, $A$, closer to the actual source plane, $a$.
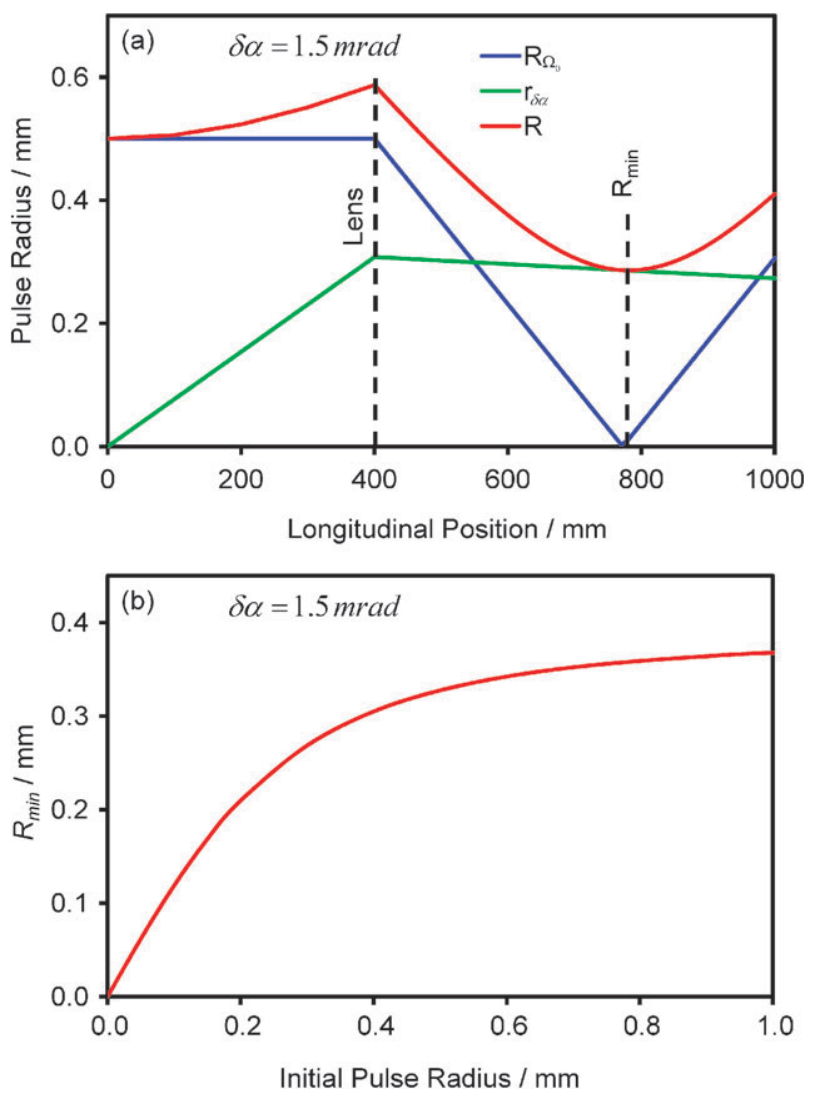

Fig. 9 Evolution of the beam waist calculated using the two-component convolution model of eqn (14) (a), and dependence of the focal spot size on the initial source dimensions (b).

Fig. $9 \mathrm{~b}$ shows how the final focus size depends on the initial source dimension. Additionally, a smaller focal size can be obtained by reducing the intrinsic divergence $\delta \alpha$ by using higher acceleration potentials. In conventional electron beam sources, a small probe size can be readily obtained by using a nanometer scale field emission tip in combination with a high acceleration voltage. ${ }^{25,27}$ In the high current limit of pulsed electron guns, however, where bunches contain thousands or possibly millions of electrons, a finite-sized source becomes a necessity due to extraction quantum efficiency of the cathode material. Therefore, the electron beam has to be given a macroscopic divergence, $\Omega_{0}$, if a small focus size is desired. Experimentally, this can be accomplished by utilizing the negative lensing effect of Coulomb repulsions (which depends on the initial charge density) and/or by inserting a diffusive lens immediately after the source to controllably induce this divergence.

For pulsed electron guns in the space-charge limit, any effort to minimize the photoelectric momentum spread is inconsequential, since generation of a sufficiently cold beam does not totally eliminate the $\delta \alpha$ component. The random and discrete nature of the electron's position within the pulse and the corresponding irregular Coulomb repulsions can also produce an intrinsic divergence for each electron. An estimation of its magnitude can be made as follows: When the 
probability of electron to be at position $\vec{r}$ is given by $P(\vec{r})$, the mean Coulomb potential energy becomes

$$
V\left(\vec{r}_{\mathrm{i}}\right)=\int \frac{1}{4 \pi \varepsilon_{0}} \frac{e^{2}}{\left|\vec{r}-\vec{r}_{\mathrm{i}}\right|} N P(\vec{r}) \mathrm{d} \vec{r}
$$

and its deviation is given by

$$
\delta V\left(\vec{r}_{\mathrm{i}}\right)=\int \frac{1}{4 \pi \varepsilon_{0}} \frac{e^{2}}{\left|\vec{r}-\vec{r}_{\mathrm{i}}\right|} \delta(N P(\vec{r})) \mathrm{d} \vec{r}
$$

which can be approximated to give

$$
\begin{aligned}
\delta V\left(\vec{r}_{\mathrm{i}}\right) & \approx \int \frac{1}{4 \pi \varepsilon_{0}} \frac{e^{2}}{\left|\vec{r}-\vec{r}_{\mathrm{i}}\right|} N \delta P(\vec{r}) \mathrm{d} \vec{r} \\
& \approx \sqrt{\left(\frac{1}{4 \pi \varepsilon_{0}} \frac{N e^{2}}{\left|\langle\vec{r}\rangle+\delta \vec{r}-\vec{r}_{\mathrm{i}}\right|}\right)^{2}-\left(\frac{1}{4 \pi \varepsilon} \frac{N e^{2}}{\left|\langle\vec{r}\rangle-\vec{r}_{\mathrm{i}}\right|}\right)^{2}} \\
& \approx \frac{1}{4 \pi \varepsilon_{0}} \frac{N e^{2}}{r_{\mathrm{i}}^{2}} \delta r
\end{aligned}
$$

Thus, $\delta V\left(\vec{r}_{i}\right)$ becomes $\sim 0.7 \mathrm{eV}$ for $r=1 \mathrm{~mm}$ and $N=10^{6}$. MC simulations confirm that, when $10^{6}$ electrons are generated within a sphere of $1 \mathrm{~mm}$ radius, an angular deviation of $\delta \alpha=0.5 \mathrm{mrad}$ develops after a few nanoseconds, which is equivalent to an initial photoelectric kinetic energy spread of $\sim 0.2 \mathrm{eV}$.

4. Spatial resolution for space-charge limited pulses. Unlike in continuous beam diffraction, a well-focused converging beam of small size is hard to achieve when using space-charge limited electron pulses as a probe. In the high charge density regime, the smallest probe size achievable is limited by the magnitude of the Coulomb repulsion among electrons. Consequently, it becomes impossible to focus the beam to a small point on the detector. However, the actual spot size of the unscattered beam is not important, as long as the Coulomb repulsions do not alter the converging electron trajectories before the pulse has passed the interaction region. Once the pulse has passed the interaction region, the scattered electrons separate from the main beam and carry the information about the specimen (encoded in the scattering angle) to the detector. Coulomb repulsions do not affect these trajectories, since the fraction of scattered electrons is small and the scattering angles are large compared to the divergence angle of the unscattered beam. This is in contrast to the imaging mode in the electron microscope, where the signal carrying electrons are focused again after the specimen. At the high peak currents in singlepulse operation, these focusing processes can lead to trajectory displacements through random scattering of the imaging electrons and, subsequently, to a stochastic reduction of the image resolution. ${ }^{28}$

To investigate a pulsed beam's spatial resolution capability, it is necessary to obtain more detailed insights into the electron bunch properties than can be obtained from the mean field model. The $\mathrm{N}$-body Monte Carlo simulation delivers the 3dimensional position and the 3-dimensional momentum vec-
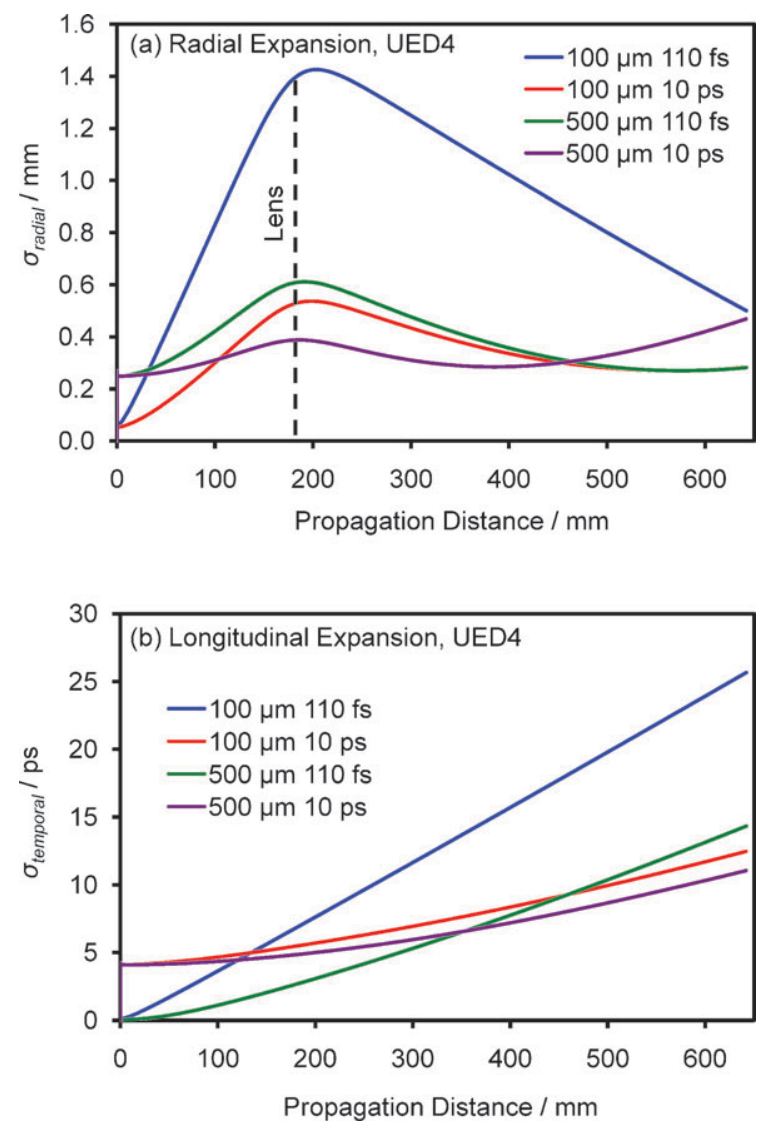

Fig. 10 Monte Carlo simulations of the radius (a) and the pulse length (b) of a bunch containing $10^{6}$ electrons using UED4 instrumental parameters.

tors for each of the particles in the bunch and is an ideal tool to study the coherence of the pulsed electron probe. We simulated electron pulses containing $10^{6}$ electrons using an arbitrary gun design for a typical UED experiment. Fig. 10a and b show the evolution of the pulse radius and duration, respectively, with four different initial conditions, which determine the extent of the initial electron packet. It is evident that a spatially and temporally confined pulse goes through an initial Coulomb explosion and expands vigorously in both the lateral and the longitudinal direction.

The lateral expansion can be compensated for by the magnetic lens, but the longitudinal expansion is unaffected by the focusing field resulting in a larger temporal extent of the pulse at later times. After the initial Coulomb explosion, the pulse expansion is approximately linear, indicating that the space-charge effect has ceased to alter the pulse expansion in the drift region. The same cannot be said for the other pulses, where the stress of high initial potential energy had been alleviated by initially stretching or expanding the excitation laser pulse in either the lateral or the longitudinal direction or both. For these pulses, the evolution of the radius and the length remains upward curved (with the exception of the lateral expansion in the region of magnetic lens focusing) for the entire drift region. All but one of the electron pulses collide with the sample as a diverging bunch, which, as was shown above, is detrimental to the spatial resolution attainable in the instrument. 
Fig. 11 shows the lateral phase space projection of the four pulses at longitudinal positions of (a) $z=100 \mathrm{~mm}$, (b) $z=300$ $\mathrm{mm}$, (c) $z=500 \mathrm{~mm}$, and (d) $z=645 \mathrm{~mm}$ (interaction region): the particles' radial divergence angle, $\kappa=\tan ^{-1}\left(p_{r} / p_{z}\right)$, defined as the angle directing the electron toward $(\kappa<0)$ or away from $(\kappa>0)$ the optical axis as a function of its radial distance from the optical axis. The initially positive, i.e. radially diverging, chirp is reversed in direction by the magnetic lens. After passing the magnetic lens, the electrons once again come in close proximity further downstream in the region of the smallest beam waist. In this region of high charge density, Coulomb repulsions gradually reverse the sign of the linear correlation between radial positions and the convergence angles until a new diverging chirp has developed. The ideal convergence angle, shown as the black line in Fig. 11d, is only reproduced by the initially well-confined pulse, since this pulse was able to expand initially and escape the sphere of influence of detrimental space-charge effects. For this pulse, $\delta \theta=1 \mathrm{mrad}$ and the resulting coherence length is $7 \AA$, using the previously stated uncertainties for the other terms in eqn (10) and (11). This particular pulse, although capable of producing high resolution information, will result in a reduced signal (for gas phase scattering), since the probability of the scattering events is directly proportional to the integrated areal density of the scattering centers, as well as the number of electrons passing through the interaction region. The signal intensity is increased, if the cross sectional area of both the electron beam and the interaction region is reduced in size (assuming the sample delivery rate is constant).

The results in Fig. 10 and 11 suggest that in order to obtain a small probe size and the corresponding convergence angles at the interaction region, the electron pulse has to avoid excessive Coulomb repulsion until the electrons have scattered from the specimen in the interaction region. Consequently, for a beam of free electrons, the high charge density has to be relieved by stretching the pulse in the longitudinal direction, such that the trajectories of the electrons are not altered by Coulomb repulsion before the interaction region and the pulse remains ideally focused. In other words, if the molecular density is low (e.g. in gas phase diffraction) or the signal averaging time is limited such that the diffraction image has to be acquired in a single shot, then good spatial resolution and good signal intensity can only come at the expense of temporal resolution. On the other hand, if the experiment can afford an intermediate number of electrons $\left(10^{3}-10^{5}\right)$, then the tradeoff between temporal and spatial resolution becomes less demanding.

To quantify these statements, we optimized instrumental parameters to obtain a smaller beam waist at the interaction region. To produce the required convergence angle, the
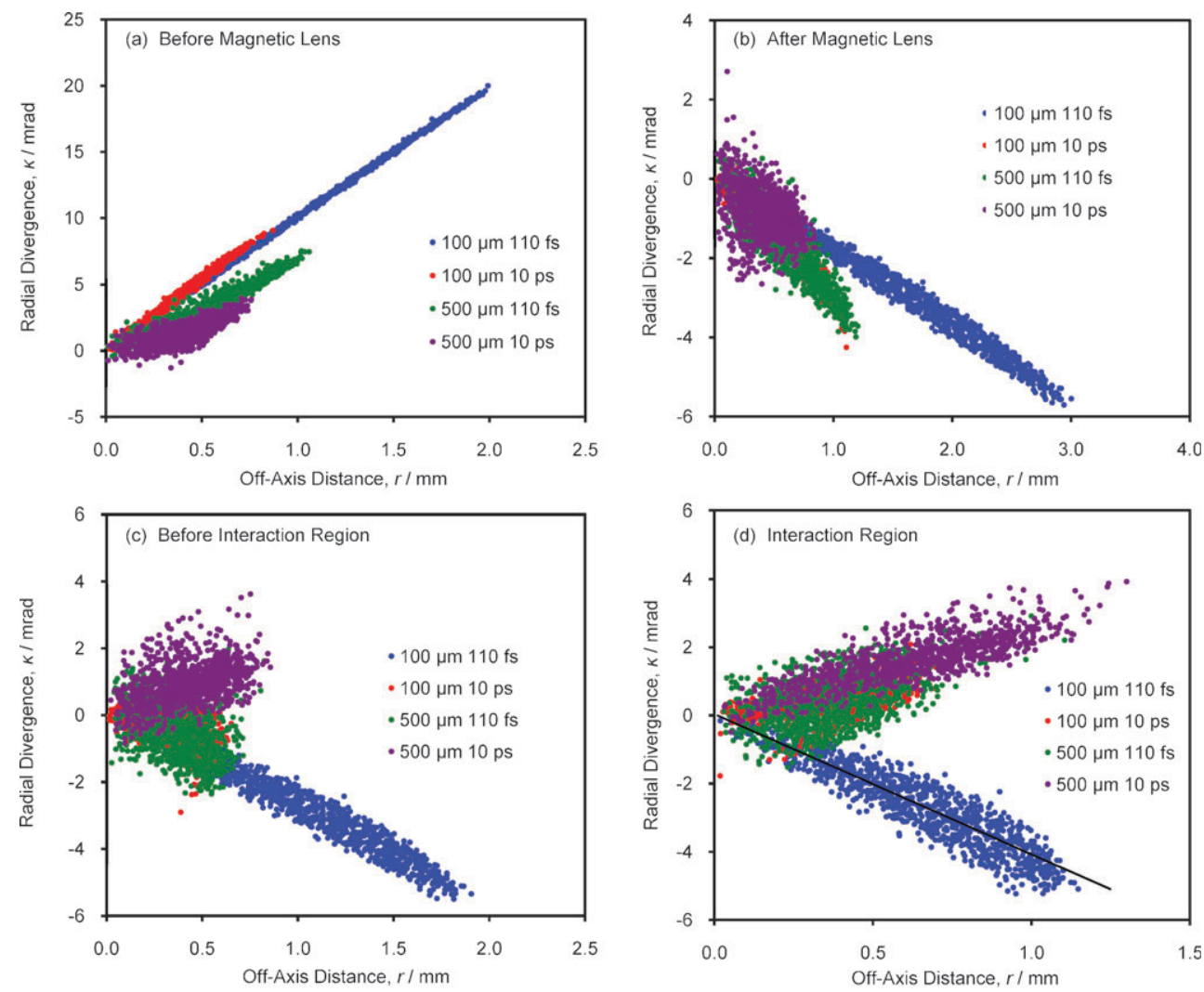

Fig. 11 Radial divergence angles of individual electrons. The results are for the pulses shown in Fig. 10 at axial distances of $z=100 \mathrm{~mm}(\mathrm{a}), z=$ $300 \mathrm{~mm}(\mathrm{~b}), z=500 \mathrm{~mm}(\mathrm{c})$, and $z=645 \mathrm{~mm}(\mathrm{~d})$. All pulses develop a diverging chirp $\left(\kappa=\tan ^{-1}\left(p_{r} / p_{z}\right)>0\right)$ due to space-charge. This linear correlation is reversed in sign $(\kappa<0)$ by the magnetic lens, a condition necessary for converging beam diffraction. However, the space-charge effect alters the converging electron trajectories for three of the four pulses in the figure by the time they arrive at the interaction region. Only the initially-confined pulse (shown in blue), which undergoes a Coulomb explosion at early times, is able to escape the sphere of influence of the space-charge effect and reproduce the ideal convergence angle (black line) in the interaction region. 


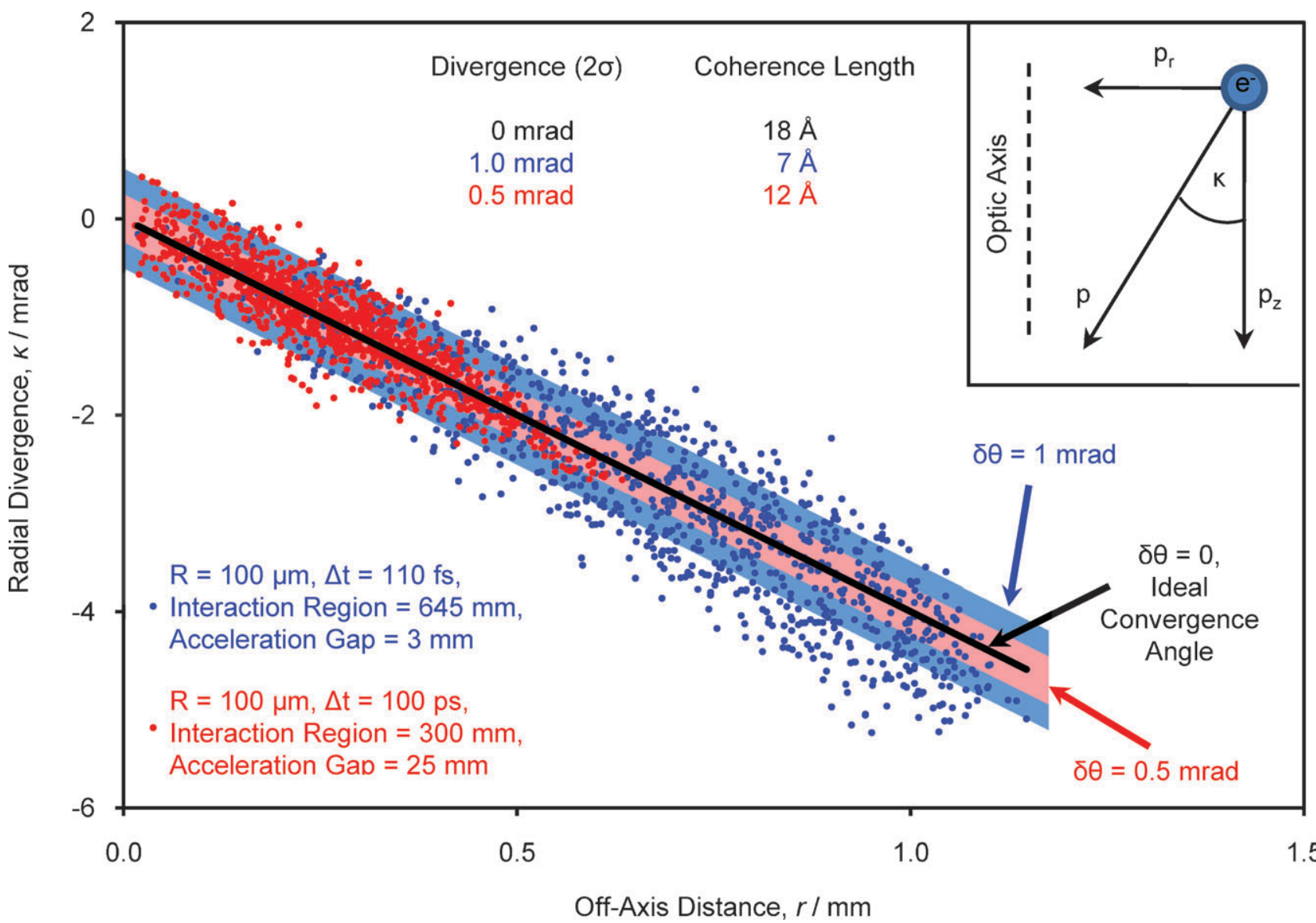

Fig. 12 Radial divergence angles of individual electrons using original (blue) and optimized (red) geometries. The uncertainty in scattering angle directly results from the deviation of incident angle from the ideal convergence angle. The red and blue shaded areas cover particles falling within an angular spread of $\pm \sigma$. The mean field theory assumes a perfect correlation between electron momenta and position, such that $\delta \theta=0$. It is evident that, in reality, electron trajectories are not perfectly correlated $(\delta \theta>0)$. The optimized instrumental geometry improves the coherence of the beam by reducing $\delta \theta$ and, additionally, gives a smaller probe size (see text).

electron beam must have expanded laterally by the time it is being focused by the magnetic lens. If, however, the pulse is initially stretched in time to say $\sim 100 \mathrm{ps}$, then, for this prolate pulse, the intrinsic rate of the lateral expansion is too small to produce a beam big enough. Experimentally, a larger beam size at the magnetic lens position can be realized in three ways: (i) by using a diverging lens in the source region, (ii) by increasing the acceleration gap, or (iii) by placing the magnetic lens farther away from the photocathode. In the last two instances, which depend on the initial charge density, the pulse is given more time to expand before it is being focused toward the interaction region, i.e. the space-charge effect is used as a diverging lens. Interestingly, a longer acceleration region must not necessarily have a detrimental effect on the temporal expansion of the electron pulse, as was previously postulated. ${ }^{29}$ Since the early events in the lifetime of the electron pulse determine its future behavior, this measure can, under certain conditions, bring about a reduction in the longitudinal momentum spread (see Appendix F).

Using the second approach to expand the beam, a pulse containing $10^{6}$ electrons giving improved spatial resolution compared to the previous pulses is shown in Fig. 12. The experimental conditions in this case are the following: A uniform lateral profile with a small initial radius $(r=100$ $\mu \mathrm{m})$, a broad Gaussian profile in time $(\Delta t=100 \mathrm{ps})$, a large acceleration gap $(d=25 \mathrm{~mm})$, a magnetic lens located at $z=$ $180 \mathrm{~mm}$, and a shorter drift space to the interaction region at $z=300 \mathrm{~mm}$. For this pulse, $\delta \theta=0.5 \mathrm{mrad}$ and the resulting coherence length is $12 \AA$, using the previously stated uncertainties for the other terms in eqn (10) and (11). Alternatively, a small probe size could also be realized, if the pulse started out with a big initial diameter. However, we found that due to spherical aberration of the magnetic lens, this approach was less successful. It should be noted that the optimized pulse shown in Fig. 12, although being better than the pulse under the original design, might not be the best pulse given the multitude of experimental parameters. The search for the optimal configuration would involve minimizing the pulse waist and the convergence angles to acceptable values, by simultaneously changing several experimental parameters, including the electron pulses initial length and width, the length of the acceleration gap, the position of the magnetic lens, and the drift length to the interaction region.

\section{Summary and conclusion}

The electron propagation dynamics determine the spatiotemporal resolution of diffraction of imaging. In this contribution, 
we address the fundamental issues involved in capturing ultrafast dynamics on the atomic scale using pulsed electrons in diffraction and microscopy. The approach involves analytical expressions and MC simulations, together with comparisons with the experimental results.

The key findings of the present study are the following. First, as the number of electrons is increased within a pulse, the space-charge effect overpowers the contribution of the initial kinetic energy distribution. The dependence on electron density, acceleration voltage, lens geometry, dispersion in electron momentum, and the propagation time, are factors that were considered here. In the absence of space-charge, the temporal extent can be obtained from eqn (1), or (2) while in the presence of space-charge, eqn (3) must be invoked with appropriate initial conditions. Both the MC simulations and the mean field theory give similar results only for longitudinal extent of pulses, as shown in Fig. 4 and 6.

Second, for space-charge limited electron bunches, the mean field theory gives a good estimation of the longitudinal spreading of the pulses, but the evolution of the pulse radius is not well predicted using this approach. The mean field theory, therefore, can give an estimate of the temporal resolution (as determined by the longitudinal extent). However, for a converging geometry (see Fig. 1 and 7), the observed at-thedetector coherence length, which is given by $1 / \delta s$ of eqn (10), is very large when considering the mean field theory, since it relies on the assumption that $\delta \theta=0$ (i.e. all electrons fall perfectly on the black line in the electron phase space diagrams of Fig. 11d and 12), and coherence is only limited by the smaller terms of eqn (10). In reality, coherence is mainly limited by the distribution of scattering angles resulting from the individual electron trajectories and its value is clear from the results of our MC simulations in Fig. 12. If the beam geometry is not converging ideally, the lateral size of the interaction region needs to be considered in eqn (10). Thus, the use of $r_{\mathrm{c}}=\lambda /(2 \pi \delta \alpha)$ by many as a measure of coherence is incomplete. We also note that, because the fraction of scattered electrons is very small, the space-charge effect can be considered absent after the interaction region.

Finally, for an electron (imaging) microscope operating in the single-electron pulsed mode or continuous-wave mode, the spatial resolution limits are the same, i.e. mainly determined by the electron source brightness and the quality of lenses. The temporal resolution is determined by the spread in the initial kinetic energy of the photoelectrons and the strength of the acceleration field. If such instruments are operated at high charge densities (single-shot mode), the evaluation of the spatial and temporal resolution has to account for the space-charge effect each time the electrons come in close proximity to each other both prior and after scattering by the specimen. ${ }^{28}$

From these studies, we now address the experimental regimes of this laboratory. For single-electron UEM, it is clear that two effects have been suppressed by this development, namely the severe influence of space-charge not only on the temporal (longitudinal) broadening, but also on the spatial (lateral) dispersion, which introduces divergence and, hence, a loss in image resolution. The kinetic energy distribution, when using a cold photocathode, is limited by the excess photon energy available above the work function, which can be tuned to be less than $0.1 \mathrm{eV},{ }^{18}$ resulting in a femtosecond pulse duration. The spatial resolution achieved in single-electron pulsed mode has reached the atomic scale in UEM2 with features resolved below $3 \AA{ }^{30}$ which is comparable to the resolution capabilities of conventional electron microscopes.

In the high charge density regime, the situation is very different. In UED4, the pulse length ranges from 1-30 ps (when using $120 \mathrm{fs}$ laser excitation) depending on the number of electrons in the pulse $\left(10^{4}-10^{6}\right)$. Using a convergent beam geometry, it is possible to determine internuclear distances up to $12 \AA$ (possibly more) with a resolution of $0.01 \AA$ in diffraction. In UEC, the temporal resolution can be increased to the femtosecond regime, when using a very low electron density per pulse and a newly implemented tilted wave-front excitation scheme. ${ }^{31,32}$

All of the above treatments are for acceleration voltages of $\leq 200 \mathrm{kV}$. At higher kinetic energies, where the electrons approach the speed of light, the space-charge effect becomes less of an issue due to relativistic effects (the effective force is attenuated as $\gamma$ (see C.4) increases and the relativistic mass increases). Recent results suggest that single-shot electron diffraction patterns can be obtained using pulses containing $10^{7}$ electrons, which were accelerated to $5.4 \mathrm{MeV} .^{33}$ It was suggested that this technique could reach sub-picosecond time resolution by utilizing the longitudinal pulse compression induced through time-dependent rf-acceleration. ${ }^{34}$ Theoretically, longitudinal focusing at lower kinetic energies $(\leq 200 \mathrm{kV})$ can also be realized, either by acceleration through a static voltage gradient ${ }^{32}$ or by rf-acceleration. ${ }^{35}$ However, care should be taken in evaluating the spatial resolution capabilities of such self-compressing electron pulses, since the space-charge effect will, at high enough charge densities, adversely affect the electron trajectories in the interaction region, thereby possibly obscuring any spatial information about the specimen.

\section{Appendix}

\section{A. Scattering signal intensity}

The probability that an incident electron is scattered by an atom is given by that atom's scattering cross section $\sigma_{\mathrm{e}}$. The scattered signal is proportional to the overlap of the electron beam with the scattering centers. Considering a pulse containing $N_{\mathrm{e}}$ electrons within an area $A_{\mathrm{e}}$ (perpendicular to the propagation direction) incident on $N_{\mathrm{a}}$ scattering centers in a volume $V_{\mathrm{a}}$, the scattering signal intensity is given by

$$
\begin{aligned}
I & =\sigma_{\mathrm{e}} \int \frac{N_{\mathrm{e}}}{A_{\mathrm{e}}} \frac{N_{\mathrm{a}}}{V_{\mathrm{a}}} \mathrm{d} V \\
& =\sigma_{\mathrm{e}} N_{\mathrm{e}} N_{\mathrm{a}} \int \rho_{\mathrm{e}}(x, y) \rho_{\mathrm{a}}(x, y, z) \mathrm{d} V
\end{aligned}
$$

where $\rho_{\mathrm{e}}$ and $\rho_{\mathrm{a}}$ are the spatial distributions of the electrons and the scattering centers, respectively. The value of $N_{\mathrm{e}}$ is assumed to remain constant throughout the interaction volume, because only a small fraction of electrons are scattered without depletion of the original density. If the distributions are assumed to be uniform, then the expression further 
simplifies to

$$
I=\sigma_{\mathrm{e}} \frac{N_{\mathrm{e}}}{A_{\mathrm{e}}} \frac{N_{\mathrm{a}}}{V_{\mathrm{a}}} \int \mathrm{d} V=\sigma_{\mathrm{e}} \frac{N_{\mathrm{e}}}{A_{\mathrm{e}}} \frac{N_{\mathrm{a}}}{V_{\mathrm{a}}} A_{\mathrm{e}} l=N_{\mathrm{e}} \rho_{\mathrm{a}} \sigma_{\mathrm{e}} l
$$

where $\rho_{\mathrm{a}}$ is now the volume density of scattering centers.

The electron scattering cross section for carbon is $\sigma_{\text {carbon, } 60}$ $\mathrm{keV}=1.21 \times 10^{-18} \mathrm{~cm}^{2},{ }^{36}$ which results in a total scattering probability per electron of $\sim 10^{-3}$ for a gas density $\rho=3 \times$ $10^{16} \mathrm{~cm}^{-3}$ (=1 torr) and $l=300 \mu \mathrm{m}$. (For comparison, the scattering cross section of X-rays, $\sigma_{\text {carbon, } 82 \mathrm{keV}}=9.17 \times 10^{-23}$ $\mathrm{cm}^{2}$ and $\sigma_{\text {carbon, } 60 \mathrm{keV}}=3.19 \times 10^{-24} \mathrm{~cm}^{2} .{ }^{37}$ ) For a diffraction pattern using pulses of $10^{4}$ electrons, ten electrons are scattered per pulse and, with a repetition rate of $1 \mathrm{kHz}, 10^{4}$ electrons are collected in one second (UED3). In UEC, the density of the specimen is much higher, such that almost all the electrons are scattered, which allows for 1-5 second exposures to collect $10^{6}$ electrons using $10^{3}$ electrons per pulse at $1 \mathrm{kHz}$. Furthermore, Bragg spots are much more localized in $s$-space, which results in an improved signal quality. In single-electron UEM, an image can be obtained, when the electron pulse train contains a total of $\sim 10^{7}$ electrons, i.e. the image is formed in $\sim 1$ second, when using a repetition rate of $80 \mathrm{MHz}$.

\section{B. Bunch dimensions and standard deviations}

For a Gaussian distribution, the full-width-half-maximum (FWHM) is given as $\sqrt{8 \ln 2} \sigma$ by solving

$$
\frac{1}{\sqrt{2 \pi} \sigma} \exp \left[-\frac{x^{2}}{2 \sigma^{2}}\right]=\frac{1}{2} \frac{1}{\sqrt{2 \pi} \sigma} \text { for } 2 x=\text { FWHM }
$$

For a 1D uniform distribution, the standard deviation is obtained from

$$
\sigma^{2}=\int_{-L / 2}^{+L / 2}(x-0)^{2} \frac{1}{L} \mathrm{~d} x=\frac{L^{2}}{12}
$$

For a 2D radial uniform distribution, the standard deviation is obtained from

$$
\sigma^{2}=\int_{-R}^{+R}(x-0)^{2} \frac{2 \sqrt{R^{2}-x^{2}}}{\pi R^{2}} \mathrm{~d} x=\frac{R^{2}}{4}
$$

The conversion relations for the length and radius of cylindrical, ellipsoidal and Gaussian pulse shapes are given in Table 1.

\section{Initial kinetic energy}

The temporal length of the laser pulse, as well it's photon energy, is directly translated onto the temporal length of the electron packet. A free electron is generated with an initial momentum of magnitude $p_{\mathrm{i}}$ at $t=0$ and then accelerated between the cathode and the anode, with are separated by a

Table 1 Relations between standard deviation $(\sigma)$ and other measured widths

\begin{tabular}{llllll}
\hline & \multicolumn{2}{c}{ Maximum-extent } & & \multicolumn{2}{l}{ Full-width-half-maximum } \\
\cline { 2 - 3 } \cline { 5 - 6 } & Length & Diameter & & Longitudinal & Lateral \\
\hline Cylindrical & $\sigma \sqrt{12}$ & $4 \sigma$ & & & $\sigma \sqrt{12}$ \\
Ellipsoidal & $\sigma \sqrt{20}$ & $\sigma \sqrt{20}$ & & $\sigma \sqrt{10}$ & $\sigma \sqrt{10}$ \\
Gaussian & & & & $\sigma \sqrt{8 \ln 2}$ & $\sigma \sqrt{8 \ln 2}$ \\
\hline
\end{tabular}

distance $d$ and held at a potential difference $V$. The electron gains momentum under the influence of the constant electric field:

$$
p(t)=p_{\mathrm{i}}+\frac{e V}{d} t
$$

The time the electron spends in the acceleration region is thus $t_{\mathrm{a}}=\frac{d}{e V}\left(p_{\mathrm{f}}-p_{\mathrm{i}}\right)$, where $p_{\mathrm{f}}$ is the electron momentum after exiting the acceleration gap. In the field-free region, the electron travels at a constant velocity $v_{\mathrm{f}}$ for a drift time $t_{\mathrm{d}}=l / v_{\mathrm{f}}$. The total flight time is then simply $t=t_{\mathrm{a}}+t_{\mathrm{d}}$.

The time spread of an ensemble of electrons with an initial momentum spread $\Delta p_{\mathrm{i}}$ will be

$$
\Delta t^{2}=\left(\frac{\partial t}{\partial p_{\mathrm{i}}}\right)^{2} \Delta p_{\mathrm{i}}^{2}
$$

To evaluate the coefficient $\partial t / \partial p_{\mathrm{i}}=\partial t_{\mathrm{a}} / \partial p_{\mathrm{i}}+\partial t_{\mathrm{d}} / \partial p_{\mathrm{i}}$ relating the momentum spread to time spread we need equations for the relativistic kinetic energy, $E$, and the relativistic momentum, $p$ :

$$
\begin{gathered}
E=\sqrt{p^{2} c^{2}+m_{0}^{2} c^{4}}-m_{0} c^{2} \\
p=\frac{m_{0} v}{\sqrt{1-\left(\frac{v}{c}\right)^{2}}}=\gamma m_{0} v
\end{gathered}
$$

and their derivatives:

$$
\begin{gathered}
\frac{\partial E}{\partial p}=\frac{p c^{2}}{\sqrt{p^{2} c^{2}+m_{0}^{2} c^{4}}}=\frac{p}{m_{0}} \frac{1}{\sqrt{\left(\frac{p}{m_{0} c}\right)^{2}+1}}=\frac{p}{m_{0}} \frac{1}{\gamma}=v \\
\frac{\partial p}{\partial v}=\frac{m_{0}}{\left[1-\left(\frac{v}{c}\right)^{2}\right]^{3 / 2}}=\gamma^{3} m_{0}
\end{gathered}
$$

Such that

$$
\begin{aligned}
\frac{\partial t_{\mathrm{a}}}{\partial p_{\mathrm{i}}} & =\left(\frac{d}{e V}\right)\left(\frac{\partial p_{\mathrm{f}}}{\partial p_{\mathrm{i}}}-1\right) \\
& =\left(\frac{d}{e V}\right)\left(\frac{\partial p_{\mathrm{f}}}{\partial E_{\mathrm{f}}} \frac{\partial E_{\mathrm{f}}}{\partial E_{\mathrm{i}}} \frac{\partial E_{\mathrm{i}}}{\partial p_{\mathrm{i}}}-1\right) \\
& =\left(\frac{d}{e V}\right)\left(\frac{v_{\mathrm{i}}}{v_{\mathrm{f}}}-1\right)
\end{aligned}
$$

upon using eqn (C.5) and $\partial E_{\mathrm{f}} / \partial E_{\mathrm{i}}=1$. Also

$$
\begin{aligned}
\frac{\partial t_{\mathrm{d}}}{\partial p_{\mathrm{i}}} & =\frac{\partial t_{\mathrm{d}}}{\partial v_{\mathrm{f}}} \frac{\partial v_{\mathrm{f}}}{\partial p_{\mathrm{f}}} \frac{\partial p_{\mathrm{f}}}{\partial p_{\mathrm{i}}} \\
& =-\frac{1}{v_{\mathrm{f}}^{2}} \frac{1}{\gamma_{\mathrm{f}}^{3} m_{0}} \frac{v_{\mathrm{i}}}{v_{\mathrm{f}}}
\end{aligned}
$$

Substituting the combined eqn (C.7) and (C.8) into eqn (C.2) and taking the square root leads to

$$
\delta t=\left|-\frac{d}{e V}\left(1-\frac{v_{\mathrm{i}}}{v_{\mathrm{f}}}\right)-\frac{l}{m_{0} \gamma_{\mathrm{f}}^{3}} \frac{v_{\mathrm{i}}}{v_{\mathrm{f}}^{3}}\right| \delta p_{\mathrm{i}}
$$




\section{Axial acceleration in uniform disk charge distribution}

For thin disk of uniform charge distribution, the Coulomb force at the axial position, $z$, can be evaluated as

$$
\begin{aligned}
F_{x}(z) & =\frac{e}{4 \pi \varepsilon_{0}} \int \mathrm{d} V \rho \frac{\vec{r}}{r^{3}} \\
& =\frac{e}{4 \pi \varepsilon_{0}} \rho \int_{0}^{R} R^{\prime} \mathrm{d} R^{\prime} \int_{0}^{2 \pi} \mathrm{d} \Theta^{\prime} \int_{-L / 2}^{L / 2} \mathrm{~d} Z^{\prime} \frac{z+Z^{\prime}}{\left(\sqrt{R^{\prime 2}+\left(z+Z^{\prime}\right)^{2}}\right)^{3}}
\end{aligned}
$$

where $R$ is the radius, $L$ is the thickness of disk, and $\rho=N e /$ $\pi R^{2} L$ is the uniform charge density. The integration yields

$$
\begin{aligned}
F_{x}(z)= & \frac{e}{4 \pi \varepsilon_{0}} \rho \pi\left(\sqrt{(L+2 z)^{2}}-\sqrt{(L-2 z)^{2}}\right. \\
& \left.+\sqrt{4 R^{2}+(L-2 z)^{2}}-\sqrt{4 R^{2}+(L+2 z)^{2}}\right)
\end{aligned}
$$

The force at the axial position $z=L / 2$ becomes

$$
\begin{aligned}
F_{x}\left(\frac{L}{2}\right) & =\frac{e}{4 \pi \varepsilon_{0}} \rho 2 \pi\left(R+L-\sqrt{R^{2}+L^{2}}\right) \\
& =\frac{e}{4 \pi \varepsilon_{0}}\left(\frac{N e}{\pi R^{2} L}\right) 2 \pi \frac{2 R L}{R+L+\sqrt{R^{2}+L^{2}}} \\
& =\frac{e}{2 \pi \varepsilon_{0}} \frac{N e}{R^{2}} \frac{2}{1+\frac{L}{R}+\sqrt{1+\left(\frac{L}{R}\right)^{2}}}
\end{aligned}
$$

And the axial acceleration becomes

$$
\frac{1}{2} \frac{\mathrm{d}^{2} L}{\mathrm{~d} t^{2}}=\frac{\mathrm{d}^{2} z}{\mathrm{~d} t^{2}}=a_{\mathrm{x}}=\frac{F_{\mathrm{x}}(z=L / 2)}{m_{e}}
$$

which is eqn (3). The result is in agreement with that of ref. 10, in which the authors integrate Poisson's equation for a charge distribution to get the axial potential. Differentiation of the potential with respect to the longitudinal coordinate gives the electric field and ultimately the acceleration in that direction.

\section{E. Attenuation of interference intensity due to incoherence}

In UED, the sinusoidal intensity is attenuated by blurring through a normally distributed $s$ as

$$
\begin{aligned}
& I(s) \propto \int \sin (x r) \frac{1}{\sqrt{2 \pi} \sigma_{s}} \exp \left[-\frac{(x-s)^{2}}{2 \sigma_{s}^{2}}\right] \mathrm{d} x=\sin (s r) \exp \left[-\frac{r^{2} \sigma_{s}^{2}}{2}\right] \\
& \exp \left[-\frac{r^{2} \sigma_{s}^{2}}{2}\right]=\frac{1}{\sqrt[s]{e}} \approx 0.88 \text { at } r=\frac{1}{2 \sigma_{s}} \\
& \exp \left[-\frac{r^{2} \sigma_{s}^{2}}{2}\right]=\frac{1}{\sqrt{e}} \approx 0.61 \text { at } r=\frac{1}{\sigma_{s}} \\
& \exp \left[-\frac{r^{2} \sigma_{s}^{2}}{2}\right]=\frac{1}{e} \approx 0.37 \text { at } r=\frac{\sqrt{2}}{\sigma_{s}} \\
& \exp \left[-\frac{r^{2} \sigma_{s}^{2}}{2}\right]=\frac{1}{e^{2}} \approx 0.14 \text { at } r=\frac{2}{\sigma_{s}}
\end{aligned}
$$

The conventional definition of coherence length corresponds to where the intensity is attenuated down to $88 \%$.
In UEC, the Bragg spot is blurred and the signal contrast is attenuated as

$$
\begin{aligned}
& I(s) \propto \sum_{i} \int \frac{1}{\sqrt{2 \pi} \sigma_{i}} \exp \left[-\frac{\left(x-s_{i}\right)^{2}}{2 \sigma_{i}^{2}}\right] \frac{1}{\sqrt{2 \pi} \sigma_{s}} \exp \left[-\frac{(x-s)^{2}}{2 \sigma_{s}^{2}}\right] \mathrm{d} x \\
& =\sum_{i} \frac{1}{\sqrt{2 \pi\left(\sigma_{s}^{2}+\sigma_{i}^{2}\right)}} \exp \left[-\frac{\left(s-s_{i}\right)^{2}}{2\left(\sigma_{s}^{2}+\sigma_{i}^{2}\right)}\right] \text { where } s_{i}=\frac{2 \pi i}{r} \\
& \text { Contrast }=\frac{I\left(s_{i}\right)-I\left(s_{i}+\frac{\pi}{r}\right)}{I\left(s_{i}\right)} \\
& =1-\frac{\sum_{i=1} 2 \exp \left[-\frac{\left(\frac{2 \pi}{r}\left(i-\frac{1}{2}\right)\right)^{2}}{2\left(\sigma_{s}^{2}+\sigma_{i}^{2}\right)}\right]}{1+\sum_{i=1} 2 \exp \left[-\frac{\left(\frac{2 \pi i}{r}\right)^{2}}{2\left(\sigma_{s}^{2}+\sigma_{i}^{2}\right)}\right]} \\
& \approx 1-2 \exp \left[-\frac{\left(\frac{\pi}{r}\right)^{2}}{2 \sigma_{s}^{2}}\right] \\
& \text { Contrast }=0.99 \text { at } r=\frac{1}{\sigma_{s}} \\
& \text { Contrast }=0.83 \text { at } r=\frac{\sqrt{2}}{\sigma_{s}} \\
& \text { Contrast }=0.43 \text { at } r=\frac{2}{\sigma_{s}} \\
& \text { Contrast }=0.00 \text { at } r=\frac{4}{\sigma_{s}}
\end{aligned}
$$

\section{F. Suppression of Coulomb repulsion during the acceleration}

Commonly efforts have been made to shorten the acceleration gap in order to accelerate the electron packet as quickly as possible to shorten their propagation time and thereby minimize the effects of the initial Coulomb explosion. This school of thought was mostly based on the concern that at the moment of generation of the photoelectrons, the charge density is unfavorably high. From the eqn (1) and (2) it is evident that the temporal spread due to initial kinetic energy spread is directly proportional to the acceleration gap, though this contribution is in the femtosecond regime. Interestingly, from the eqn (3) it can be deduced that in the case of a very thin disk, the initial thickness does not alter the magnitude of Coulomb repulsion, since the aspect ratio, $L / R$, is vanishingly small and remains small for early times. Therefore, the Coulomb repulsion can be treated as an approximately constant acceleration in the beginning. It can be shown using the mean field theory that the space-charge induced momentum spreads of both freely drifting electrons and accelerating electrons are the same after traveling the same distance. To illustrate this point, we separately consider the mechanical work done on the front and the rear end of the pulse, by the acceleration field and Coulomb repulsion. In the thin-disk limit we have

$$
\begin{aligned}
& W_{F}=m\left(a_{\mathrm{A}}+a_{\mathrm{X}}\right) d \\
& W_{B}=m\left(a_{\mathrm{A}}-a_{\mathrm{X}}\right) d
\end{aligned}
$$


This work results in changes of the kinetic energies according to

$$
\begin{aligned}
& W_{F}=E_{\mathrm{f}, F}-E_{\mathrm{i}, F} \\
& W_{B}=E_{\mathrm{f}, B}-E_{\mathrm{i}, B}
\end{aligned}
$$

For acceleration, $E_{\mathrm{i}}=0$ and $m a_{\mathrm{A}} d=e V$, while for a free drifting, $E_{\mathrm{i}}=e V$ and $a_{\mathrm{A}}=0$. Either way, the final kinetic energies are

$$
\begin{aligned}
& E_{\mathrm{f}, F}=e V+m a_{\mathrm{X}} d \\
& E_{\mathrm{f}, B}=e V-m a_{\mathrm{X}} d
\end{aligned}
$$

Therefore, they both result in an energy spread of $2 m a_{X} d$, or a momentum spread

$$
\begin{aligned}
\Delta p & =\frac{m}{p} \Delta E=\frac{m}{\sqrt{2 m E}} \Delta E=\sqrt{\frac{m}{2 E}} \Delta E \\
& =\sqrt{\frac{m}{2 e V}} 2 m a_{\mathrm{X}} d=\sqrt{\frac{2 m^{3}}{e V}} a_{\mathrm{X}} d
\end{aligned}
$$

We note that it takes accelerating electrons twice as long to travel the same distance. Also it can be shown that the spatial expansion of the accelerating electrons is four times larger than of free flying electrons after traveling the same distance, resulting in a four times larger increase in the temporal spread.

$$
\begin{aligned}
& L_{\text {accelerating }}(x=d)=L_{\mathrm{i}}+2 a_{\mathrm{X}}\left(\frac{2 d}{v_{\mathrm{i}}+v_{\mathrm{f}}}\right)^{2} \approx 8 a_{X}\left(\frac{d}{v_{\mathrm{f}}}\right)^{2} \\
& L_{\text {drifting }}(x=d)=L_{\mathrm{i}}+2 a_{\mathrm{X}}\left(\frac{d}{v_{\mathrm{f}}}\right)^{2} \approx 2 a_{X}\left(\frac{d}{v_{\mathrm{f}}}\right)^{2} \\
& R_{\text {accelerating }}(x=d)=R_{\mathrm{i}}+a_{\mathrm{R}}\left(\frac{2 d}{v_{\mathrm{i}}+v_{\mathrm{f}}}\right)^{2} \approx R_{\mathrm{i}} \\
& R_{\text {drifting }}(x=d)=R_{\mathrm{i}}+a_{\mathrm{R}}\left(\frac{d}{v_{\mathrm{f}}}\right)^{2} \approx R_{\mathrm{i}}
\end{aligned}
$$

However, in reality, the expanding diameter will reduce the Coulomb repulsions and therefore the momentum spread can be smaller, at the cost of a larger beam size.

\section{References}

1 A. H. Zewail, Annu. Rev. Phys. Chem., 2006, 57, 65-103, and references therein.

2 J. C. Williamson, M. Dantus, S. B. Kim and A. H. Zewail, Chem. Phys. Lett., 1992, 196, 529-534; H. Ihee, V. A. Lobastov, U. M. Gomez, B. M. Goodson, R. Srinivasan, C. Y. Ruan and A. H. Zewail, Science, 2001, 291, 458-462.

3 C. Y. Ruan, F. Vigliotti, V. A. Lobastov, S. Y. Chen and A. H. Zewail, Proc. Natl. Acad. Sci. U. S. A., 2004, 101, 1123-1128.

4 C. Y. Ruan, V. A. Lobastov, F. Vigliotti, S. Y. Chen and A. H. Zewail, Science, 2004, 304, 80-84.

5 V. A. Lobastov, R. Srinivasan and A. H. Zewail, Proc. Natl. Acad. Sci. U. S. A., 2005, 102, 7069-7073.

6 J. C. Williamson and A. H. Zewail, Chem. Phys. Lett., 1993, 209, 10-16; M. Dantus, S. B. Kim, J. C. Williamson and A. H. Zewail, J. Phys. Chem., 1994, 98, 2782-2796.

7 B. W. Reed, J. Appl. Phys., 2006, 100, 034916.

8 S. Collin, M. Merano, M. Gatri, S. Sonderegger, P. Renucci, J. D. Ganiere and B. Deveaud, J. Appl. Phys., 2005, 98, 094910.
9 M. Merano, S. Collin, P. Renucci, M. Gatri, S. Sonderegger, A. Crottini, J. D. Ganiere and B. Deveaud, Rev. Sci. Instrum., 2005, 76, 085108 .

10 B. L. Qian and H. E. Elsayed-Ali, J. Appl. Phys., 2003, 94, 803-806.

11 B. J. Siwick, J. R. Dwyer, R. E. Jordan and R. J. D. Miller, J. Appl. Phys., 2002, 92, 1643-1648.

12 J. Orloff, Handbook of Charged Particle Optics, CRC Press, New York, 1997G. H. Jansen, Coulomb Interaction in Particle Beams, Academic Press, Inc., San Diego, 1990.

13 O. J. Luiten, S. B. van der Geer, M. J. de Loos, F. B. Kiewiet and M. J. van der Wiel, Phys. Rev. Lett., 2004, 93, 094802.

14 W. E. King, G. H. Campbell, A. Frank, B. Reed, J. F. Schmerge, B. J. Siwick, B. C. Stuart and P. M. Weber, J. Appl. Phys., 2005, 97, 111101.

15 A. M. Michalik and J. E. Sipe, J. Appl. Phys., 2006, 99, 054908.

16 B. Barwick, C. Corder, J. Strohaber, N. Chandler-Smith, C. Uiterwaal and H. Batelaan, New J. Phys., 2007, 9, 142.

17 J. G. Endriz and W. E. Spicer, Phys. Rev. Lett., 1971, 27, 570-573.

18 A. Janzen, B. Krenzer, O. Heinz, P. Zhou, D. Thien, A. Hanisch, F. Heringdorf, D. von der Linde and M. H. von Hoegen, Rev. Sci. Instrum., 2007, 78, 013906.

19 J. R. Dwyer, R. E. Jordan, C. T. Hebeisen, M. Harb, R. Ernstorfer, T. Dartigalongue and R. J. D. Miller, J. Modern Opt., 2007, 54, 923-942.

20 R. Srinivasan, V. A. Lobastov, C. Y. Ruan and A. H. Zewail, Helv. Chim. Acta, 2003, 86, 1763-1838.

21 M. Born and E. Wolf, Principles of Optics, Pergamon Press, Oxford, 1975

22 J. Rosenzweig, G. Travish and L. Serafini, The Physics and Applications of High Brightness Electron Beams, World Scientific, Singapore, 2003.

23 S. T. Park, J. S. Feenstra and A. H. Zewail, J. Chem. Phys., 2006, 124, 174707.

24 D. S. Yang, N. Gedik and A. H. Zewail, J. Phys. Chem. C, 2007, 111, 4889-4919.

25 L. Reimer, Transmission Electron Microscopy, Springer, Berlin, 1997.

26 J. C. Cowley, Diffraction Physics, Elsevier, Amsterdam, 1995I. Hargittai and M. Hargittai, Stereochemical Applications of Gas-Phase Electron Diffraction, VCH Publishers Inc, New York, 1988.

27 A. Tonomura, Electron Holography, Springer, Berlin, 1999.

28 M. R. Armstrong, K. Boyden, N. D. Browning, G. H. Campbell, J. D. Colvin, W. J. DeHope, A. M. Frank, D. J. Gibson, F. Hartemann, J. S. Kim, W. E. King, T. B. LaGrange, B. J. Pyke, B. W. Reed, R. M. Shuttlesworth, B. C. Stuart and B. R. Torralva, Ultramicroscopy, 2007, 107, 356-367; M. R. Armstrong, B. W. Reed, B. R. Torralva and N. D. Browning, Appl. Phys. Lett., 2007, 90, 114101.

29 M. Aeschlimann, E. Hull, J. Cao, C. A. Schmuttenmaer, L. G. Jahn, Y. Gao, H. E. Elsayedali, D. A. Mantell and M. R. Scheinfein, Rev. Sci. Instrum., 1995, 66, 1000-1009.

30 H. S. Park, J. S. Baskin, O. H. Kwon and A. H. Zewail, Nano Lett., 2007, 7, 2545-2551; recent experiments showed a spatial resolution of $<3 \AA$.

31 P. Baum, D. S. Yang and A. H. Zewail, Science, 2007, 318, 788-792.

32 P. Baum and A. H. Zewail, Proc. Natl. Acad. Sci. U. S. A., 2006, 103, 16105-16110.

33 J. B. Hastings, F. M. Rudakov, D. H. Dowell, J. F. Schmerge, J. D. Cardoza, J. M. Castro, S. M. Gierman, H. Loos and P. M. Weber, Appl. Phys. Lett., 2006, 89, 184109.

34 X. J. Wang, D. Xiang, T. K. Kim and H. Ihee, J. Korean Phys. Soc., 2006, 48, 390-396.

35 T. van Oudheusden, E. F. de Jong, S. B. van der Geer, W. Root, O. J. Luiten and B. J. Siwick, J. Appl. Phys., 2007, 102, 093501; L. Veisz, G. Kurkin, K. Chernov, V. Tarnetsky, A. Apolonski, F. Krausz and E. Fill, New J. Phys., 2007, 9, 451.

36 F. Salvat and A. Jablonski, NIST Electron Elastic-Scattering Cross-Section Database: Version 3.1, National Institute for Standards and Technology, 2002.

37 M. J. Berger, J. H. Hubbell, S. M. Seltzer, J. Chang, J. S. Coursey, R. Sukumar and D. S. Zucker, XCOM: Photon Cross Section Database (version 1.3), National Institute for Standards and Technology, 2005.

38 V. A. Lobastov, J. Weissenrieder, J. Tang and A. H. Zewail, NanoLett., 2007, 7, 2552-2558. 